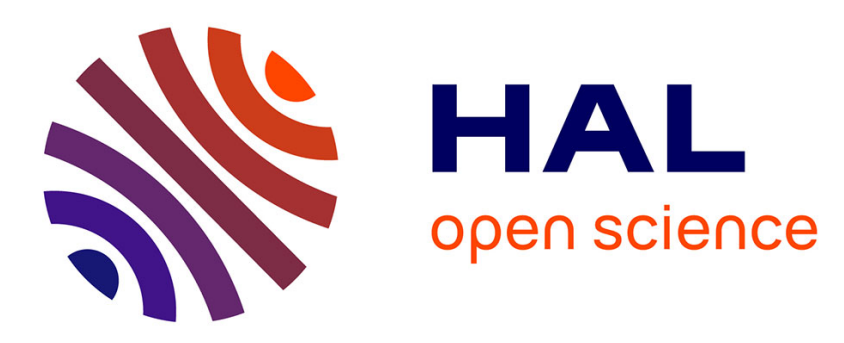

\title{
Influence of wind on extreme wave events: Experimental and numerical approaches
}

\author{
Christian Kharif, Jean-Paul Giovanangeli, Julien Touboul, Laurent Grare, \\ Efim Pelinovsky
}

\section{- To cite this version:}

Christian Kharif, Jean-Paul Giovanangeli, Julien Touboul, Laurent Grare, Efim Pelinovsky. Influence of wind on extreme wave events: Experimental and numerical approaches. Journal of Fluid Mechanics, 2008, 594, pp.209-247. 10.1017/S0022112007009019 . hal-00159430v2

\section{HAL Id: hal-00159430 \\ https://hal.science/hal-00159430v2}

Submitted on 7 Sep 2007

HAL is a multi-disciplinary open access archive for the deposit and dissemination of scientific research documents, whether they are published or not. The documents may come from teaching and research institutions in France or abroad, or from public or private research centers.
L'archive ouverte pluridisciplinaire HAL, est destinée au dépôt et à la diffusion de documents scientifiques de niveau recherche, publiés ou non, émanant des établissements d'enseignement et de recherche français ou étrangers, des laboratoires publics ou privés. 


\title{
Influence of wind on extreme wave events: Experimental and numerical approaches
}

\author{
By C. KHAR IF ${ }^{1} \dagger$, J. - P. G I O VA NA N G E L I ${ }^{1}$, \\ J. TOUBO UL ${ }^{1}$, L. GRARE \\ AND E. PELINOVSK Y \\ ${ }^{1}$ Institut de Recherche sur les Phénomènes Hors Equilibre, Aix-Marseille University, France \\ ${ }^{2}$ Institute of Applied Physics, Nizhny Novgorod, Russia.
}

(Received ?? and in revised form ??)

The influence of wind on extreme wave events in deep water is investigated experimentally and numerically. A series of experiments conducted in the Large Air-Sea Interactions Facility (LASIF-Marseille, France) shows that wind blowing over a short wave group due to the dispersive focusing of a longer frequency modulated wave train (chirped wave packet) may increase the time duration of the extreme wave event by delaying the defocusing stage. A detailed analysis of the experimental results suggests that extreme wave events may be sustained longer by the air flow separation occurring on the leeward side of the steep crests. Furthermore it is found that the frequency downshifting observed during the formation of the extreme wave event is more important when the wind velocity is larger. These experiments have pointed out that the transfer of momentum and energy is strongly increased during extreme wave events.

Two series of numerical simulations have been performed using a pressure distribution over the steep crests given by the Jeffreys'sheltering theory. The first series corresponding to the dispersive focusing confirms the experimental results. The second series that corresponds to extreme wave events due to modulational instability, shows that wind sustains steep waves which then evolve into breaking waves. Furthermore, it was shown numerically that during extreme wave events the wind-driven current could play a significant role in their persistence.

\section{Introduction}

The main objective of this paper is to better understand the physics of extreme wave events in the presence of wind. This study deals with the fundamental problem of the air flow structure above steep water wave groups and its impact on wind-wave coupling, namely its effects on air-sea fluxes. The present experimental and numerical investigations concern the rogue wave phenomenon in the presence of wind. This work which has been motivated primarily by the problem of rogue waves goes beyond the scope of these water waves and can be applied to the field of the interaction between wind and strongly modulated surface wave groups in deep water.

There are a number of physical mechanisms that focus the wave energy into a small area and produce the occurrence of extreme waves called freak or rogue waves. These events can be due to refraction (presence of variable currents or bottom topography), dispersion (frequency modulation), wave instability (the Benjamin-Feir instability also called 
modulational instability), soliton interactions, etc. For more details on these different mechanisms see the reviews on freak waves by Dysthe (2001) and Kharif \& Pelinovsky (2003). Until now there is no consensus about a unique definition of rogue wave events. One definition often used is based on height criterion. A wave is considered to be a rogue wave if its height, $H_{f}$, satisfies the condition

$$
H_{f}>2.2 H_{s} .
$$

where $H_{s}$ is the significant height.

To our knowledge, the present experimental and numerical study is the first one to consider the direct effect of strong wind on the rogue wave formation. In different situations, several authors have investigated experimentally the influence of wind on the evolution of mechanically generated gravity water waves. Bliven et al. (1986), Li et al. (1987) and Waseda \& Tulin (1999) have studied the influence of wind on Benjamin-Feir instability. Contrary to results reported by Bliven et al and Li et al, Waseda \& Tulin found that wind did not suppress the sideband instability. Banner \& Song (2002) have studied numerically the onset of wave breaking in nonlinear wave groups in presence of wind forcing. In the present paper we investigate how wind forcing modifies unforced extreme wave events due to spatio-temporal focusing and modulational instability.

Extreme wave events that are due to spatio-temporal focusing phenomenon can be described as follows. If initially short wave packets are located in front of longer wave packets having larger group velocities, then during the stage of evolution, longer waves will overtake shorter waves. A large-amplitude wave can occur at some fixed time because of superposition of all the waves merging at a given location (the focus point). Afterwards, the longer waves will be in front of the shorter waves, and the amplitude of the wave train will decrease. This focusing-defocusing cycle was described by Pelinovsky et al. (2000) within the framework of the shallow water theory. Later, Slunyaev et al (2002) used the Davey-Stewartson system for three-dimensional water waves propagating in finite depth. More recently, this technique was also used in the experiments on extreme waves conducted by Giovanangeli et al. (2005) and Touboul et al. (2006).

Another mechanism generating extreme wave events is the modulational instability or the Benjamin-Feir instability. Due to this instability uniform wave trains suffer modulationdemodulation cycles (the Fermi-Pasta-Ulam recurrence). At the maximum of modulation a frequency downshifting is observed and very steep waves occur. Many authors have investigated rogue waves or extreme wave events due to modulational instability. Among them one may cite: Henderson et al. (1999), Dysthe \& Trulsen (1999), Osborne et al. (2000), Kharif et al. (2001), Calini \& Schober (2002), Janssen (2003), Dyachenko \& Zakharov (2005) and Clamond et al. (2006). Nevertheless, all these studies did not consider the direct effect of wind on the dynamics of extreme wave events.

In presence of wind, separation of the air flow occurring in the lee of very steep crests, suggests that the Jeffreys' sheltering mechanism can be applied locally in space and time. Banner \& Melville (1976) explored both experimentally and analytically the occurrence of air-flow separation over a simple gravity surface wave. Herein we used the simple wind modelling suggested by Jeffreys (1925).

The wind influence on extreme wave events due to spatio-temporal focusing is investigated experimentally and numerically while extreme wave event caused by modulation instability is considered numerically only.

In section 2 we present the experimental facility and results concerning extreme waves generated through the spatio-temporal focusing. A wind modelling is proposed in section 3 , based on the Jeffreys' sheltering mechanism that is used for the numerical simula- 


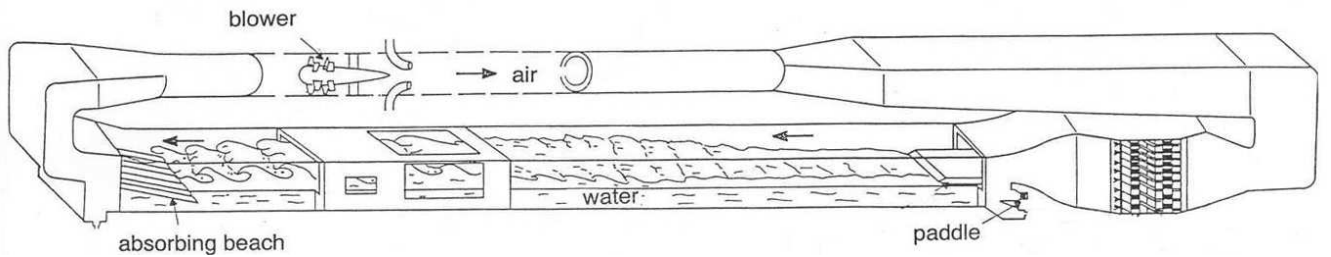

Figure 1. A schematic description of the Large Air-Sea Interactions Facility.

tions corresponding to the spatio-temporal focusing and the nonlinear focusing due to the Benjamin-Feir instability respectively.

\section{Experiments and results}

\subsection{Experimental facility}

The experiments have been conducted in the large wind-wave tank of IRPHE at Marseille Luminy. Figure 1 gives a schematic presentation of the facility. It consists of a closed loop wind tunnel located over a water tank $40 \mathrm{~m}$ long, $1 \mathrm{~m}$ deep and $2.6 \mathrm{~m}$ wide. The wind tunnel over the water flow is $40 \mathrm{~m}$ long, $3.2 \mathrm{~m}$ wide and $1.6 \mathrm{~m}$ high. The blower allows to produce wind velocities up to $14 \mathrm{~m} / \mathrm{s}$ and a computer-controlled wave maker submerged under the upstream beach can generate regular or random waves in a frequency range from $0.5 \mathrm{~Hz}$ to $2 \mathrm{~Hz}$. Particular attention has been paid to simulate pure logarithmic mean wind velocity profile with constant shear layer over the water surface. A trolley installed in the test section allows to locate probes at different fetches all along the facility. The water surface elevation is measured by using three capacitive wave gauges of $0.3 \mathrm{~mm}$ outer diameter with DANTEC model 55E capacitance measuring units. A wave gauge is located at a fixed fetch of $1 \mathrm{~m}$ from the upstream beach. The other wave gauges are installed on the trolley to measure the water surface elevation at different fetches from the upstream beach. The typical sensitivity of the wave probes is of order $0.6 \mathrm{~V} / \mathrm{cm}$. The longitudinal and vertical air flow velocity fluctuations, $u^{\prime}$ and $w^{\prime}$, have been measured by means of an $X$-hot wire. The fetch is defined as the distance between the probes on the trolley and the end of the upstream beach where air flow meets the water surface.

\subsection{The spatio-temporal focusing mechanism}

Extreme wave events are generated by means of a spatio-temporal focusing mechanism. This mechanism is based upon the dispersive behavior of water waves. In this chirped wave packet, the leading waves have a higher frequency than trailing waves. Within the framework of a linear approach of the problem the sea surface is a superposition of linear waves of frequencies $\omega(x, t)$. According to Whitham (1967), the spatio-temporal evolution of the frequency of these components is governed by the following hyperbolic equation

$$
\frac{\partial \omega}{\partial t}+c_{g}(\omega) \frac{\partial \omega}{\partial x}=0
$$

where $c_{g}$ is the group velocity. This equation can be solved by using the method of characteristics. The solution is given by

$$
\omega(x, t)=\omega_{0}(\tau), \quad v_{g}(\tau)=c_{g}\left(\omega_{0}(\tau)\right) \quad \text { on } \quad t=\tau+x / v_{g}(\tau)
$$


where $\omega_{0}$ corresponds to the temporal frequency distribution of the wave train at $x=0$. The temporal partial derivative of the frequency is

$$
\frac{\partial \omega}{\partial t}=\frac{\frac{d \omega_{0}}{d \tau}}{1-\frac{x}{v_{g}^{2}} \frac{d v_{g}}{d \tau}}
$$

One can notice that the case $d v_{g} / d \tau>0$, which corresponds to short waves emitted before longer waves, leads to a singularity. This singularity corresponds to the focusing of several waves at $t=T_{f_{t h}}$ and $x=X_{f_{t h}}$. For infinite depth, the frequency to impose to the wavemaker located at $x=0$ is given by

$$
\omega(0, t)=\frac{g}{2} \frac{T_{f_{t h}}-t}{X_{f_{t h}}}
$$

where $X_{f_{t h}}$ and $T_{f_{t h}}$ are the coordinates of the point of focus in the $(x-t)$ plane. Using $\omega=2 \pi f$ the coordinates of the focus point reads

$$
\begin{aligned}
T_{f_{t h}} & =\Delta T \frac{f_{\max }}{f_{\max }-f_{\min }} \\
X_{f_{t h}} & =\frac{g \Delta T}{4 \pi} \frac{1}{f_{\max }-f_{\min }}
\end{aligned}
$$

where $f_{\max }$ and $f_{\text {min }}$ are the maximal and minimal values of the frequency imposed to the wavemaker during a period of time equal to $\Delta T$ and $g$ is the acceleration due to gravity.

The wave amplitude, $a$, satisfies the following equation

$$
\frac{\partial a^{2}}{\partial t}+\frac{\partial}{\partial x}\left(c_{g} a^{2}\right)=0
$$

This equation corresponds to the conservation of wave energy, and its solution is found explicitly by

$$
a(x, t)=\frac{a_{0}(\tau)}{\sqrt{1-\frac{x}{v_{g}^{2}} \frac{d v_{g}}{d \tau}}},
$$

where $a_{0}(\tau)$ is the temporal distribution of the wave amplitude at $x=0$. Within the framework of the linear theory, focus points are singular points where the amplitude becomes infinite and behaves like $\left(X_{f_{t h}}-x\right)^{-1 / 2}$.

Experimentally, the values $f_{\max }=1.3 \mathrm{~Hz}$ and $f_{\min }=0.8 \mathrm{~Hz}$ correspond to the maximal and minimal frequencies of the wavemaker and $\Delta T=10 \mathrm{~s}$ is the duration of the wave generation. The surface elevation given by the probe located at $1 \mathrm{~m}$ from the upstream beach is presented in Figure 2. From these data we find that $T_{f_{t h}}=26 \mathrm{~s}$ and $X_{f_{t h}}=17 \mathrm{~m}$ while the experimental values are $T_{f_{e x p}}=26 \mathrm{~s}$ and $X_{f_{e x p}}=20 \mathrm{~m}$ (see Figure 3 ). Experimental data are in close agreement with the linear theory. The difference observed between the theoretical and experimental values of $X_{f}$ is mainly due to the nonlinearity of the experimental wave train. The wave train generated at the wavemaker is uniform in amplitude, hence the short waves are more nonlinear than the longer waves, and the result is a downstream shift of the focusing location.

\subsection{Experimental results}

The focusing experiments are performed with and without wind. The same initial wave train is generated and propagated without wind, and under the action of wind for several values of the wind velocity equal to $U=4 \mathrm{~m} / \mathrm{s}, 5 \mathrm{~m} / \mathrm{s}, 6 \mathrm{~m} / \mathrm{s}$, and $8 \mathrm{~m} / \mathrm{s}$ respectively. When 


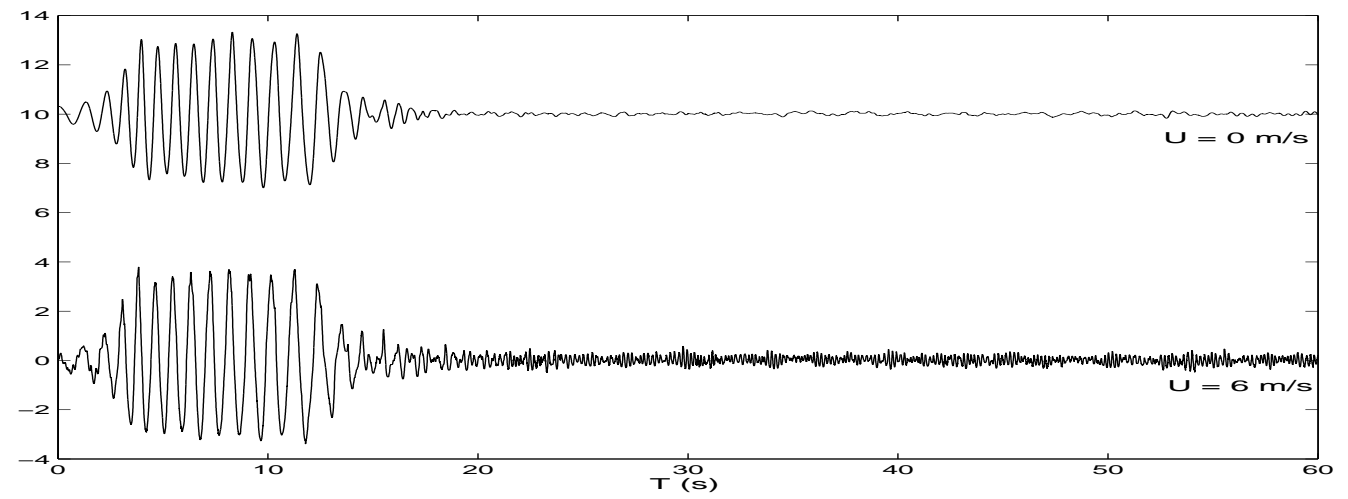

Figure 2. Surface elevation (in $\mathrm{cm}$ ) at fetch $X=1 \mathrm{~m}$, for wind speeds $U=0$ and $6 \mathrm{~m} / \mathrm{s}$ (note that for $U=0 \mathrm{~m} / \mathrm{s}$, the origin of the elevation corresponds to the value $10 \mathrm{~cm}$ ).

\begin{tabular}{c|c|}
\hline Wind Velocity $(\mathrm{m} / \mathrm{s})$ & $\left|\sqrt{<\eta^{2}>}(\mathrm{cm})\right|$ \\
\hline 0 & 1.88 \\
4 & 1.88 \\
5 & 1.87 \\
6 & 1.88 \\
8 & 1.87 \\
10 & 1.88 \\
\hline
\end{tabular}

TABLE 1. The r.m.s. elevation for different values of the wind velocity at fetch $1 \mathrm{~m}$.

the wind blows, the focusing wave train is generated once wind waves have developed. For each value of the mean wind velocity $U$ the water surface elevation is measured at $1 \mathrm{~m}$ fetch and at different fetches between $3 \mathrm{~m}$ and $35 \mathrm{~m}$. The wavemaker is driven by an analog electronic signal to produce this signal linearly varying with time from $1.3 \mathrm{~Hz}$ to $0.8 \mathrm{~Hz}$ in $10 \mathrm{~s}$, with an almost constant amplitude of the displacement. The wavemaker is totally submerged to avoid any perturbation of the air flow which could be induced by its displacement.

To ensure the repeatability of the experimental conditions under the wind action, the water elevations at $1 \mathrm{~m}$ were recorded with and without wind.

Figure 2 shows two time series of this probe, recorded with no wind, and under a wind speed $U=6 \mathrm{~m} / \mathrm{s}$. The probe record corresponding to a wind velocity equal to $6 \mathrm{~m} / \mathrm{s}$ is artificially increased by $10 \mathrm{~cm}$, for more clarity of the figure. We see that the two signals are very similar, since frequency properties, phases and duration are maintained. Some weak differences in amplitude are locally observed. Table 1 shows the root mean square of the elevation $\eta(x, t)$ obtained at fetch $1 \mathrm{~m}$ for different wind speeds. It is clear from these data that no significant variations are observed, and the experiment is considered to be repeatable in presence of wind. Results of these experiments are presented in the following subsection.

Figure 3 presents the time series of the water surface elevation $\eta(x, t)$ at different fetches for $U=0 \mathrm{~m} / \mathrm{s}$. For sake of clarity, as it has been done for Figure 2, the probes records given here are recursively increased by $10 \mathrm{~cm}$. As predicted by the linear theory 


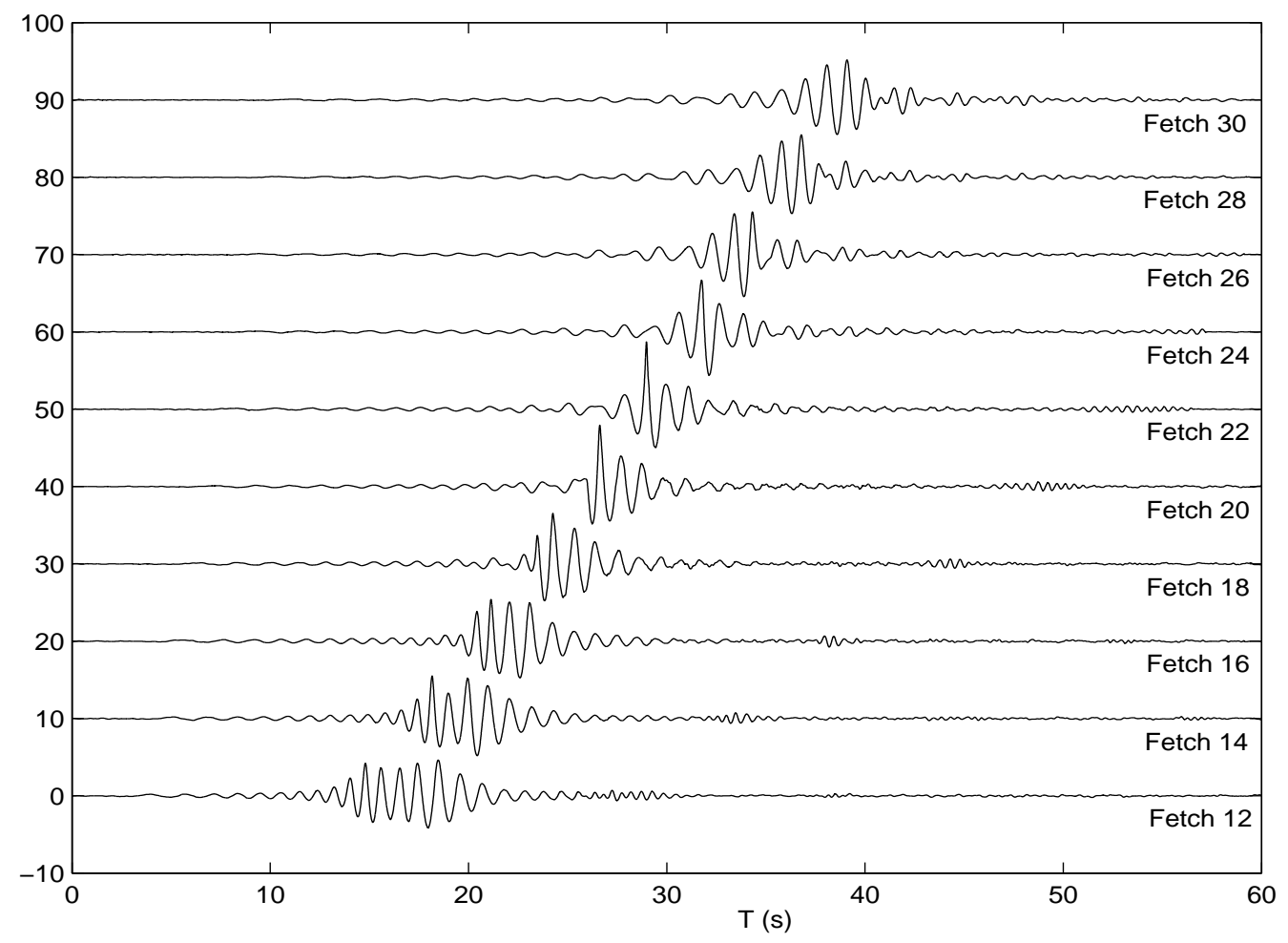

FiguRE 3. Surface elevation (in $\mathrm{cm}$ ) at several fetches (in $\mathrm{m}$ ), for wind speed $U=0 \mathrm{~m} / \mathrm{s}$, as a function of time.

of free deep water waves (no wind), dispersion leads short waves to propagate slower than long waves, and as a result, the waves focus at a given position in the wave tank leading to the occurrence of a large amplitude wave. Downstream the point of focus, the amplitude of the group decreases rapidly (defocusing).

Figure 4 shows the same time series of $\eta(x, t)$, at several values of the fetch $x$, and for a wind speed $U=6 \mathrm{~m} / \mathrm{s}$. The wave groups mechanically generated by the wavemaker are identical to those used in the experiments without wind (see Figure 2). Whatever, some differences appear in the time-space evolution of the focusing wave train. One can observe that the group of the extreme wave event is sustained longer.

For each value of the wind velocity, the amplification factor $A(x, U)$ of the group between fetches $x$ and $1 m$ can be defined as

$$
A(x, U)=\frac{H_{\max }(x, U)}{H_{r e f}}
$$

where $H_{\max }(x, U)$ is the maximal height between two consecutive crest and trough in the transient group. The height, $H_{r e f}$, of the quasi uniform wave train generated at the entrance of the tank is measured at $1 \mathrm{~m}$. The mean height crest to through is $H_{r e f}=$ $6.13 \mathrm{~cm}$.

Figure 5 gives this amplification factor as a function of the distance from the upstream beach for various values of the wind velocity, equal to $0 \mathrm{~m} / \mathrm{s}, 4 \mathrm{~m} / \mathrm{s}$ and $6 \mathrm{~m} / \mathrm{s}$. This figure shows that the effect of the wind is twofold: (i) it increases weakly the amplification factor, and (ii) it shifts downstream the focus point. Moreover, contrary to the case without wind, an asymmetry appears between focusing and defocusing stages. The slope 


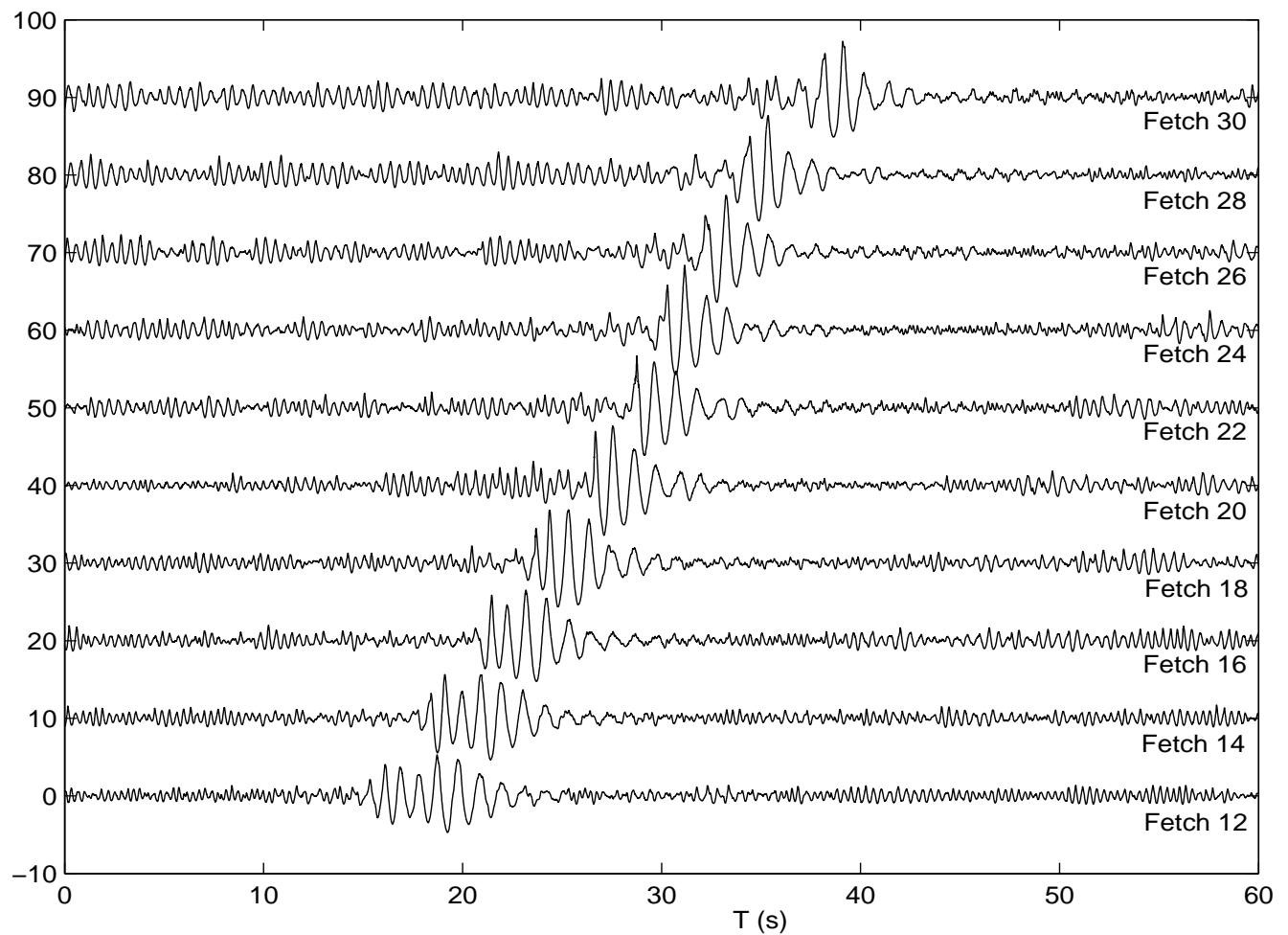

FiguRE 4. Surface elevation (in $\mathrm{cm}$ ) at several fetches (in $\mathrm{m}$ ), for wind speed $U=6 \mathrm{~m} / \mathrm{s}$, as a function of time.

of the curves corresponding to defocusing changes. Before the focus point, it is interesting to notice that the wind has no effect on the amplification factor. One can observe that the rogue wave criterion $(A>2.2)$ is satisfied for a longer period of time. The effect of the wind on the rogue wave is to shift the focusing point downstream, and to increase slightly its amplitude. It is also interesting to emphasize that the rogue wave criterion is satisfied for a longer distance, while the wind velocity increases.

To better understand the time-space evolution of the wave group with and without wind, the time series are analyzed by means of a wavelet analysis. Figure 6 displays the local wavelet power spectra of probe records at several fetches, without wind. The wavelet power spectrum is defined as the square of the modulus of the wavelet transform. These spectra show the time-frequency evolution of the wave group as it propagates downstream the wave tank. At short fetches, the waves of high frequencies are in front of the group and the waves of lower frequencies at its back. As it propagates downstream, focusing and defocusing processes are observed. The focus point corresponds to the merging of all the frequencies. Downstream the focus point, the waves of low frequencies are in front of the group, and the high frequencies at its back

Figure 7 shows the local wavelet power spectra of probe records at the same fetches, for a wind speed of $6 \mathrm{~m} / \mathrm{s}$. Contrary to the case without wind, the focusing point is shifted downstream the wave tank, confirming what we observe in Figure 5. We note that the coherence of the group is maintained longer and consequently the extreme wave event mechanism is sustained longer. This could explain the asymmetry observed in the amplification curves.

We observe in Figures 4 and 7, that the background wind waves are suppressed by the 


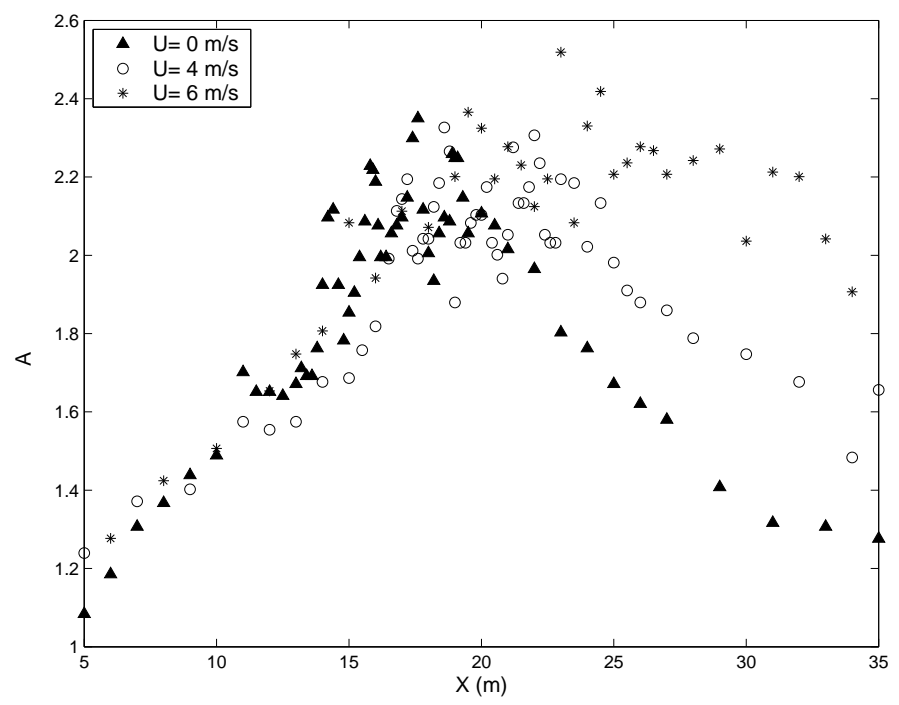

FiguRE 5. Evolution of the amplification factor $A(x, U)$ as a function of the distance, for several values of the wind speed.

extreme wave event. The phenomenon of high frequency waves suppressed by strongly nonlinear low frequency waves has been investigated by Balk (1996). He showed that the effect of the long wave is to transport the short wave action to high wave numbers, where high dissipation occurs.

To summarize the main experimental results we can claim that the effect of wind on the extreme wave event mechanism is to shift the focus point downstream, to increase its amplitude and lifetime leading to an asymmetry of the amplification curve. Figures 6 and 7 demonstrate that the effect of the wind is to transform the short group containing the extreme wave into a long-lived short group. The effect of the wind is to delay the defocusing stage.

\subsection{Wind-wave coupling over focusing group}

The previous results show that in presence of wind the focusing/defocusing phenomenon is significantly modified. The focus point is shifted downstream, the amplitude and duration of the extreme wave event are increased even for weak values of wind velocity. To clarify the physical processes which could explain these results, a second series of experiments has been conducted to investigate the wind-wave interaction during the focusing and defocusing stages.

The experimental conditions are similar to those described previously except that other probes have been installed on the trolley to measure pressure and velocity fluctuations in the air flow at different heights in the turbulent boundary layer and different fetches in the wave tank. The longitudinal and vertical wind speed fluctuations, $u^{\prime}$ and $w^{\prime}$, are measured by means of an X-wire mounted on two DANTEC model constant-temperature anemometers. The two hot wires of the $\mathrm{X}$-wire have been calibrated before and after the experiments in a small wind tunnel. A least-square regression law is used to relate the output voltages of each anemometer to the effective cooling velocities $U_{\text {eff } 1}$ and $U_{\text {eff } 2}$ respectively for the wires $i=1,2$, using the Collis and Williams law

$$
E_{i}^{2}=A_{i}+B_{i} U_{\text {effi }}^{n_{i}}, \quad i=1,2
$$

where the effective velocities $U_{\text {effi }}$ are related to the wind speed by the following rela- 

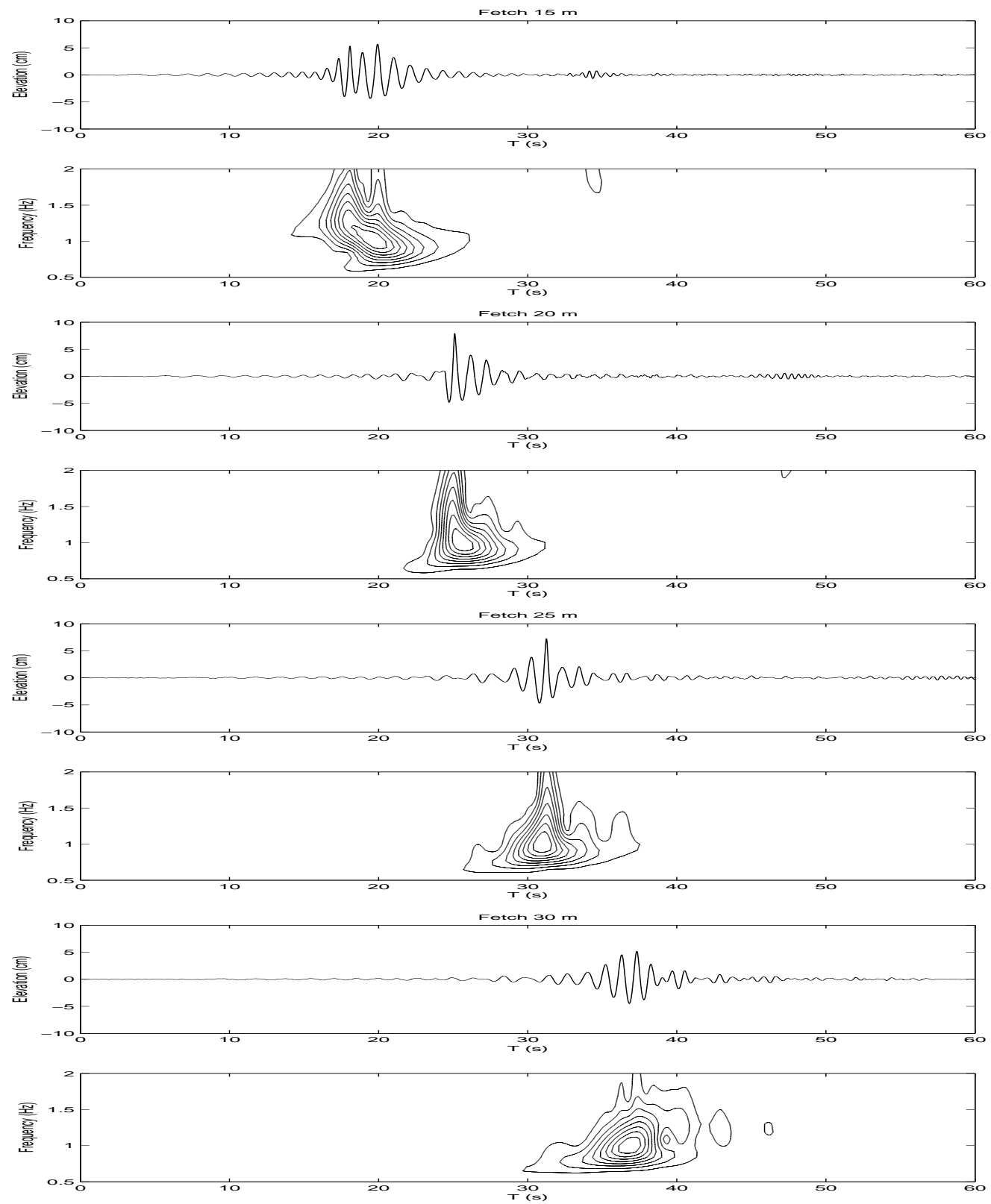

FigURE 6. Local wavelet power spectra of probe records at fetches $x=15,20,25$ and $30 \mathrm{~m}$ for a wind speed value of $U=0 \mathrm{~m} / \mathrm{s}$. The vertical and bottom axes are the frequency and time respectively.

tionship

$$
U_{\text {eff } i}=\sqrt{\cos ^{2} \Phi_{i}+K_{i}^{2} \sin ^{2} \Phi_{i}} \quad i=1,2
$$

Here $K_{i}$ is the cooling factor of wire $i$ and $\Phi_{i}$ is the angle between the wind speed vector and the normal to wire $i$. The coefficients $A_{i}, B_{i}$ and $n_{i}$ are computed during the calibration. The two components $u^{\prime}$ and $w^{\prime}$ of the wind velocity are determined from the ratio $E_{1} / E_{2}$. 

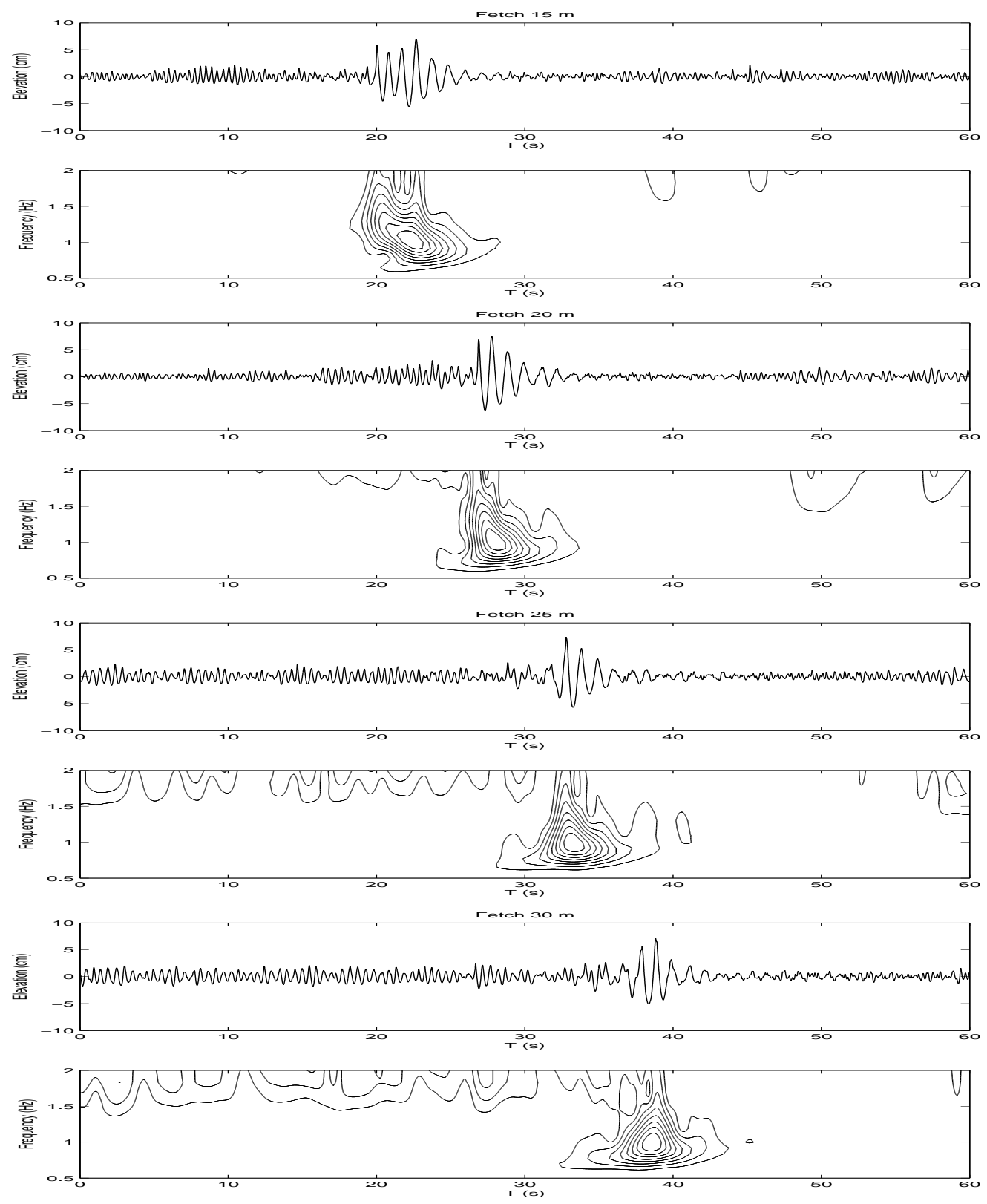

FiguRE 7. Local wavelet power spectra of probe records at fetches $x=15,20,25$ and $30 \mathrm{~m}$ for a wind speed value of $U=6 \mathrm{~m} / \mathrm{s}$. The vertical and bottom axes are the frequency and time respectively.

The pressure fluctuations in the air flow are measured using a method developed by Giovanangeli (1988) whereby the static pressure is determined from the difference between the observed total pressure and the dynamical pressure derived from the velocity measurements. The total pressure is measured using a bleed-type pressure sensor TSI model 1412J. Details about the method and features of the pressure probe can be found in the paper by Giovanangeli \& Chambaud (1987). It was shown that the pressure probe in combination with the method used here allows measurements of the static pressure 


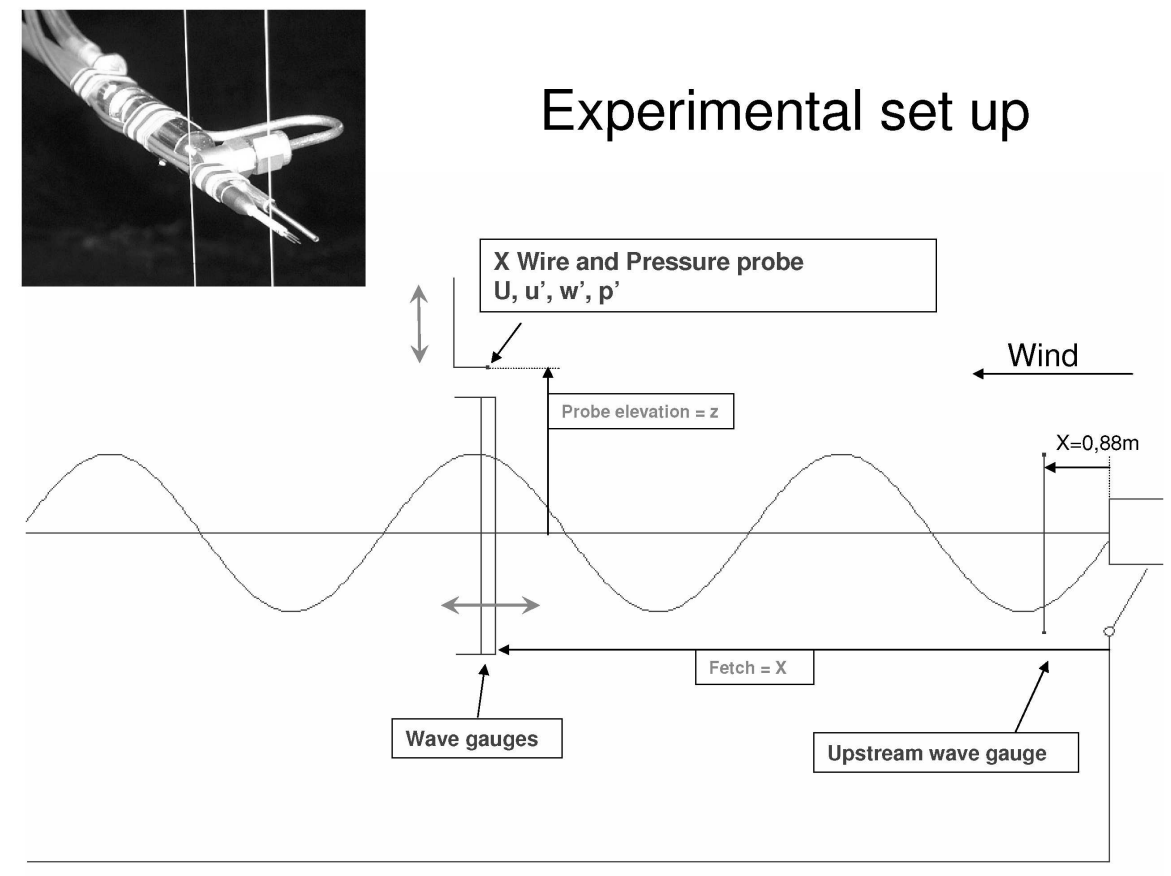

FiguRE 8. A schematic representation of the experimental set-up.

fluctuations in the air flow, particularly close to steep surface waves, with an accuracy of $0.05 P a$.

The key point of the present experiments is to measure the static pressure fluctuations in the presence of paddle waves. As proved by others [Latif (1974), Papadimitrakis et al. (1986) and Banner (1990)] the driving mechanism and the displacements of the wavemaker induce rather large acoustic pressure fluctuations inside the wave tank. Hence, they used different methods to correct this effect. Rather than trying to correct the contamination of the acoustic mode, we choose to avoid this effect by recording the wavemaker displacements and analyzing the data only when it is turned off. Since acoustic pressure fluctuations propagate at the sound velocity we record output voltage of the probes without acoustic contamination. The procedure summarized herein is described in detail by Mastenbroeck et al. (1996).

The amplitude and longitudinal wave slope are computed by means of two wave gauges installed on the trolley and $2 \mathrm{~cm}$ spatially separated in the mean wind direction. Figure 8 gives a schematic representation of the experimental set-up installed on the trolley. Figure 9 shows the time series of the water surface elevation $\eta$ in $\mathrm{cm}$, the total vertical momentum flux from wind to water waves $\left\langle u^{\prime} w^{\prime}\right\rangle$, the form drag $\left\langle p^{\prime} \partial \eta / \partial x\right\rangle$ and energy flux $<p^{\prime} \partial \eta / \partial t>$ from wind to water waves. The pressure fluctuation is $p^{\prime}, \partial \eta / \partial x$ is the longitudinal wave slope and $\partial \eta / \partial t$ is the time derivative of the surface elevation. The form drag, momentum and energy fluxes are time averaged on an interval of 2 seconds. For a wind velocity $U=6 \mathrm{~m} / \mathrm{s}$, at fetch $20 \mathrm{~m}$ and height of $13 \mathrm{~cm}$ above the mean water level, it can be observed that the occurrence of focusing wave groups corresponds to a significant enhancement of the fluxes. Notice that the time origin corresponds to the occurrence of the extreme wave event. Note that the air flow pressure fluctuations $p^{\prime}$ were 

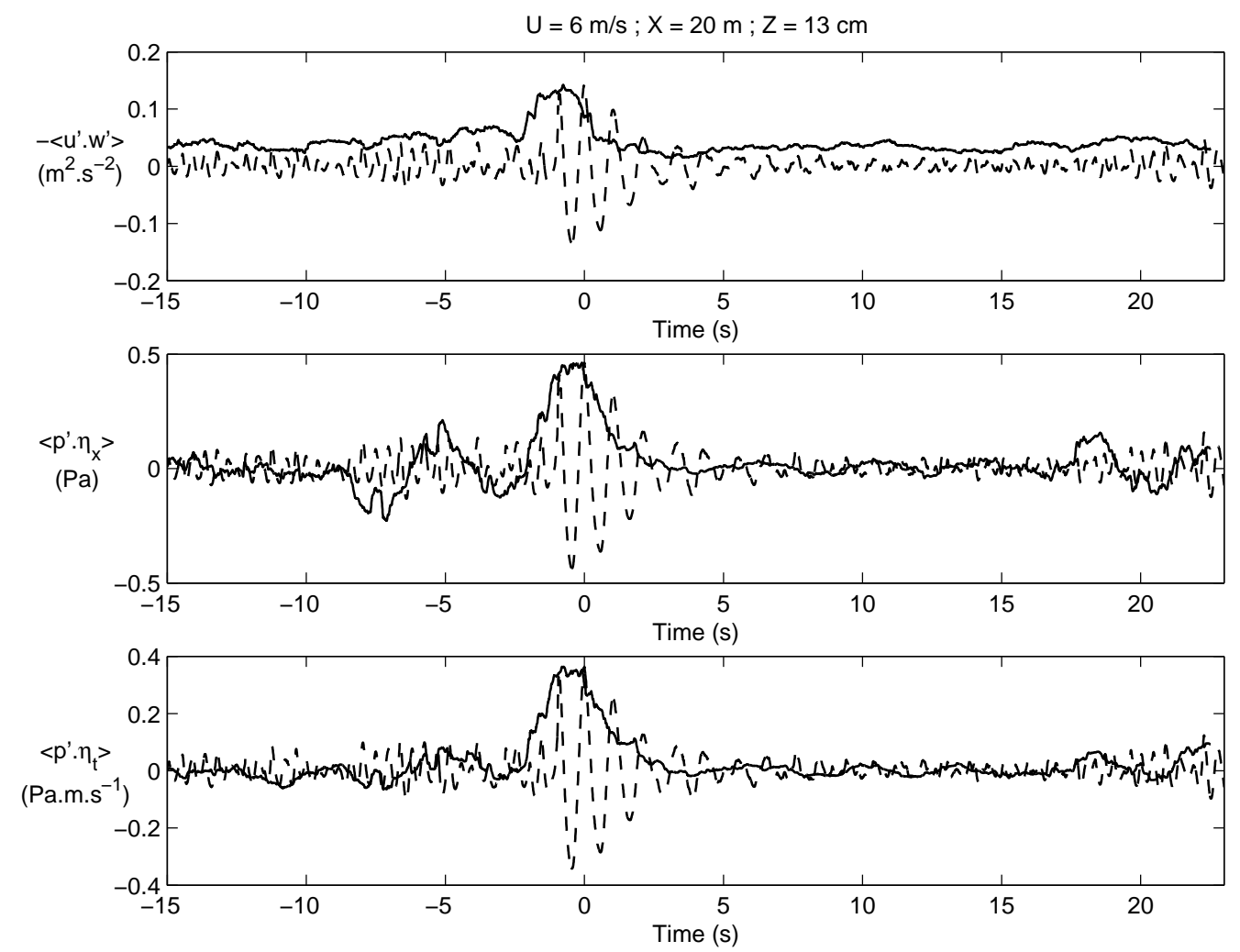

Figure 9. Time series of the surface elevation $\eta$ (dashed lines), vertical momentum flux (solid line in the top panel), form drag (solid line in the middle panel) and energy flux (solid line in the bottom panel).

measured at different heights above the interface. Hence, it was not possible to determine the exact value of the form $\mathrm{drag}\left\langle p^{\prime} \partial \eta / \partial x>\right.$ at $z=\eta(t)$. However the determination of $<p^{\prime} \partial \eta / \partial x>$ at the height $z$ will provide crucial information about wind wave coupling between the air flow and the interface during the focusing event.

Local wavelet power spectra of the surface elevation has been computed and as shown in Figure 7 the duration of the extreme wave event is increased in presence of wind.

Figures 10 and 11 correspond to the local wavelet power spectra of the longitudinal wind velocity fluctuation $u^{\prime}$ and pressure fluctuation $p^{\prime}$ along the wave tank, at height $z=13 \mathrm{~cm}$ above the mean water level, for mean wind velocity $U=6 \mathrm{~cm} / \mathrm{s}$. From these figures it is not easy to observe the coupling between the group and the turbulent boundary layer. This is mainly due to the broad-band character of the spectra.

To emphasize this coupling, a cross-wavelet analysis has been applied between $u^{\prime}$ and $w^{\prime}, p^{\prime}$ and $\partial \eta / \partial x$ and, $p^{\prime}$ and $\partial \eta / \partial t$ respectively (for more detail see Torrence \& Compo (1998)). These terms are considered as a contribution in time and frequency range to the total stress, form drag and energy flux from wind to waves respectively. Figure 12 shows the cross-wavelet power for $u^{\prime}$ and $w^{\prime}$. The cross-wavelet spectrum for the longitudinal and transversal velocity fluctuations is defined as the product of the wavelet transform of $u^{\prime}$ and complex conjugate wavelet transform of $w^{\prime}$. The cross-wavelet power is the modulus of the cross-wavelet spectrum. For more details see the practical stepby-step guide to wavelet analysis by Torrence \& Compo (1998). A strong correlation 


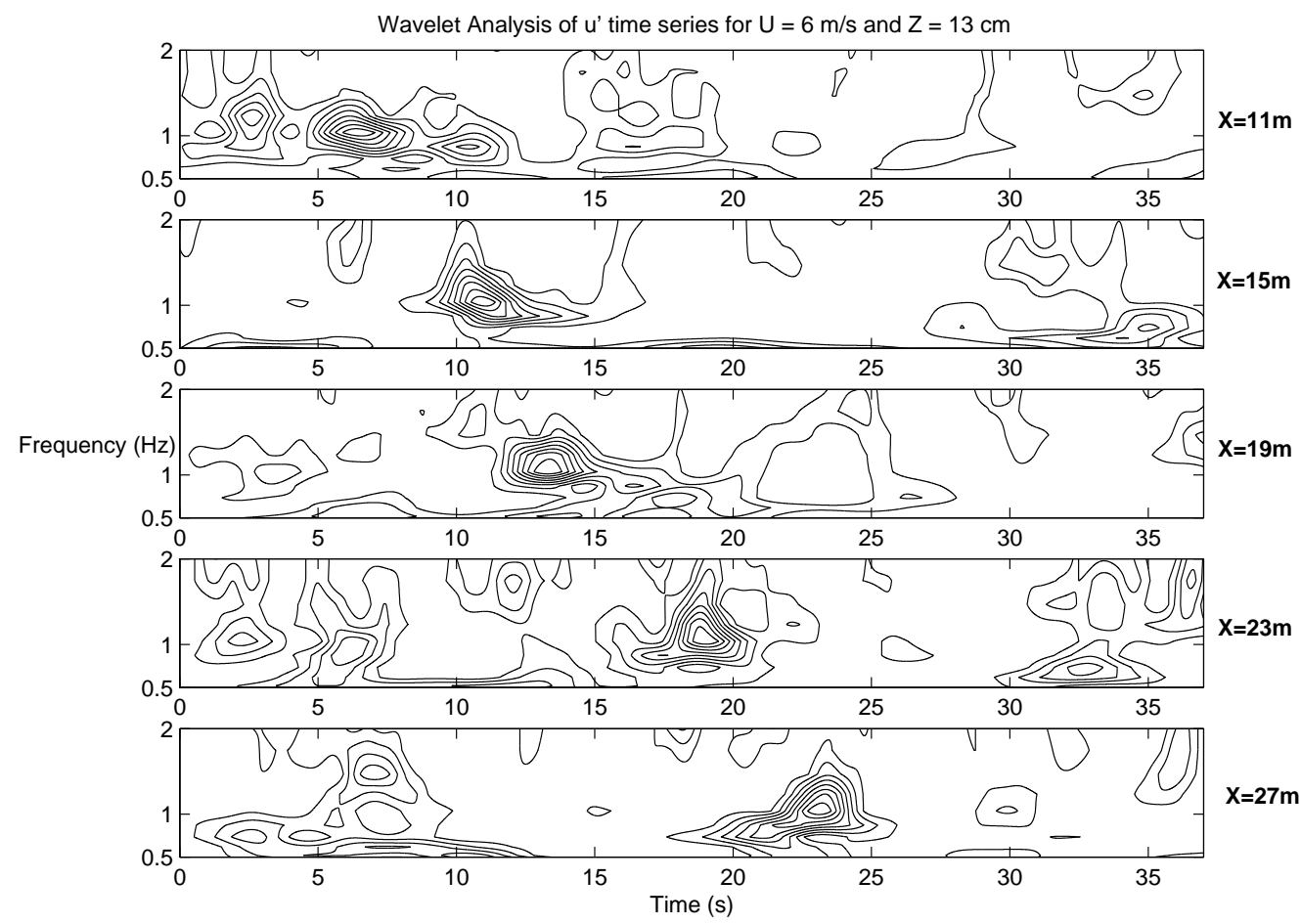

FigURE 10. Local wavelet power spectra of the longitudinal wind velocity fluctuations, $u^{\prime}$, at several fetches for a mean wind velocity $U=6 \mathrm{~m} / \mathrm{s}$ and $13 \mathrm{~cm}$ above the mean water level. The vertical and bottom axes are the frequency and time respectively.

between $u^{\prime}$ and $w^{\prime}$ is observed above the groups. At fetch $x=11 m$, two groups can be seen, the higher frequencies components propagate in front of the lower frequencies components. At fetch $x=17 m$ the two groups have begun to merge into one group which propagates downstream. We can observe that the maximum of the cross-wavelet power travels downstream with the group.

Figures 13 and 14 confirm the behavior observed above and demonstrate the strong correlation existing between the group and the form drag and the energy transfer from wind to water waves. Air-sea fluxes are strongly enhanced in presence of strongly modulated wave groups.

An accurate measurement of the maximum of the wavelet power spectrum of the surface elevation $\eta$ is calculated. Figure 15 displays the characteristic curves of this maximum for several values of the wind velocity. The figure shows that the maximum propagates downstream with a constant velocity which increases as the wind speed increases. This velocity is equal to $0.87 \mathrm{~m} / \mathrm{s}, 0.90 \mathrm{~m} / \mathrm{s}, 0.92 \mathrm{~m} / \mathrm{s}$ and $0.93 \mathrm{~m} / \mathrm{s}$ for $U=0 \mathrm{~m} / \mathrm{s}, 4 \mathrm{~m} / \mathrm{s}, 6 \mathrm{~m} / \mathrm{s}$ and $8 \mathrm{~m} / \mathrm{s}$ respectively. These values which are equal to the slope of the characteristic curves plotted in Figure 15 correspond to mean values of the group velocity in the vicinity of the focus area. For $U=0 \mathrm{~m} / \mathrm{s}$, a more careful inspection shows fluctuations of the group velocity during extreme wave event as observed numerically by ? at the maximum of modulation due to Benjamin-Feir instability. The distance between two consecutive probes is too large to detect the group velocity fluctuations accurately.

In Figure 16 are plotted the characteristic curves corresponding to the maximum of the cross-wavelet power for $u^{\prime}$ and $w^{\prime}$ at several altitude above the mean water surface from $z=13 \mathrm{~cm}$ to $z=30 \mathrm{~cm}$, for $U=6 \mathrm{~m} / \mathrm{s}$. We can notice that this maximum travels 


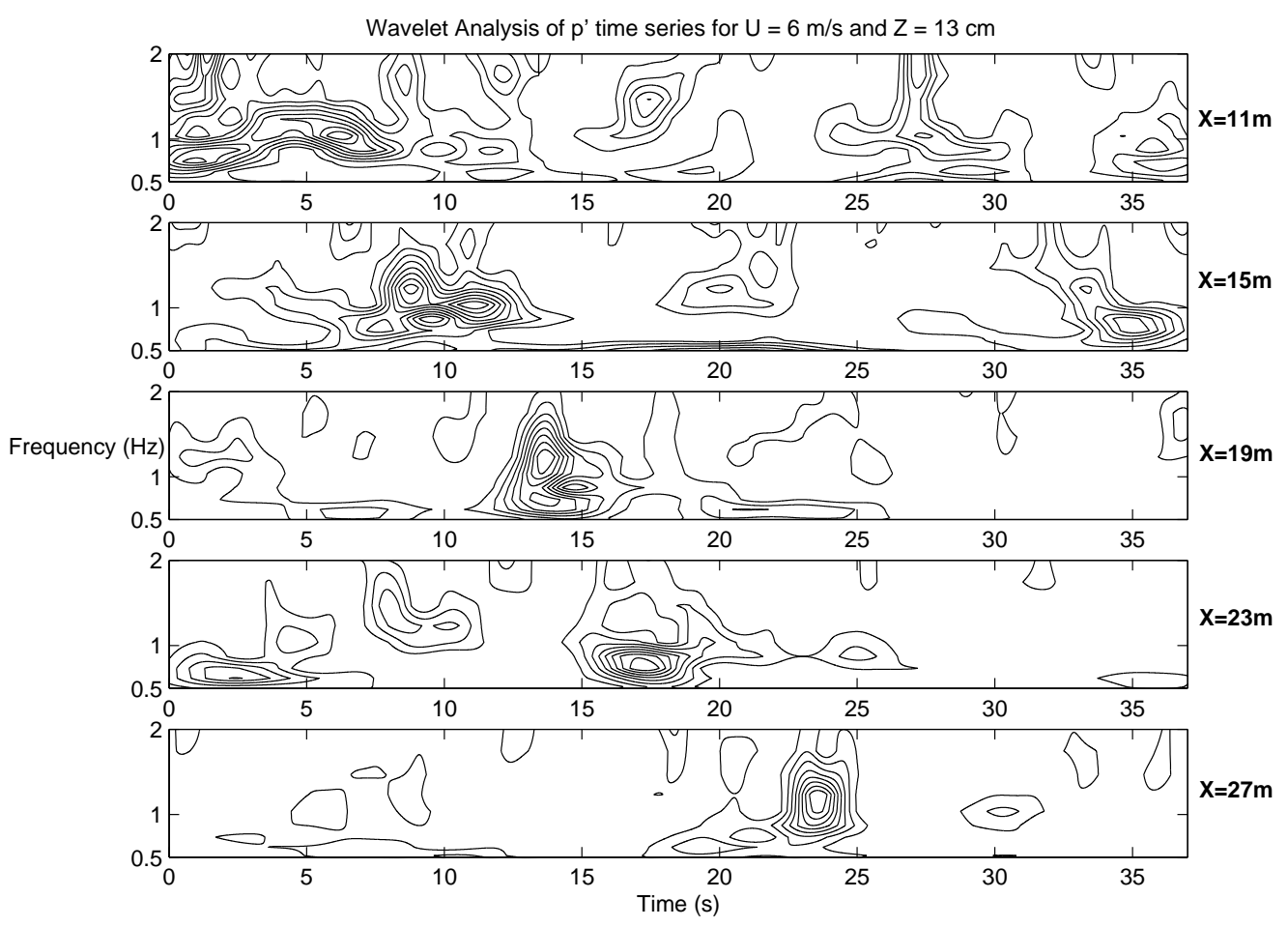

FigURE 11. Local wavelet power spectra of the pressure fluctuations, $p^{\prime}$, at several fetches for a mean wind velocity $U=6 \mathrm{~m} / \mathrm{s}$ and $13 \mathrm{~cm}$ above the mean water level. The vertical and bottom axes are the frequency and time respectively.

at a speed close to the velocity defined previously, independently of the altitude $z$ above the mean water level. This figure emphasizes that the coupling between the air flow and the water wave group is effective in the whole boundary layer and strongly attached to the group.

In Figure 17 are plotted the characteristic curves corresponding to the maximum of the cross-wavelet power for $u^{\prime}$ and $w^{\prime}$ at the altitude $z=14 \mathrm{~cm}$ above the mean water level for $U=4 \mathrm{~m} / \mathrm{s}, 6 \mathrm{~m} / \mathrm{s}$ and $8 \mathrm{~m} / \mathrm{s}$ respectively. The space-time diagram shows that this maximum propagates at a velocity close to the velocity of the maximum of the wavelet power spectrum of the surface elevation.

In Figure 18 are plotted the characteristic curves corresponding to the maximum of the cross-wavelet power of $u^{\prime}$ and $\partial \eta / \partial t$ at several altitude above the mean water surface from $z=13 \mathrm{~cm}$ to $z=30 \mathrm{~cm}$, for $U=6 \mathrm{~m} / \mathrm{s}$. Herein again this maximum corresponding to the transfer of energy between wind and waves propagates with the velocity of the maximum of the wavelet power spectrum of the surface elevation. As for the maximum of the cross-wavelet power for $u^{\prime}$ and $w^{\prime}$ corresponding the momentum flux, the maximum of cross-wavelet power corresponding to the instantaneous flux of energy to waves due to pressure fluctuations above the group, $p^{\prime} \partial \eta / \partial t$, travels downstream at the velocity of the maximum of the cross-wavelet power spectrum of the surface elevation.

Figure 19 shows the spatial evolution of the frequency corresponding to the maximum of the cross-wavelet power spectrum of the surface elevation as a function of $x$ for $11 m<x<29 m$, i.e. in the vicinity of the focus point for several values of the wind velocity. It can be seen that the frequency decreases during the formation of the extreme wave event. Hence rogue wave is associated with frequency downshifting. This feature 


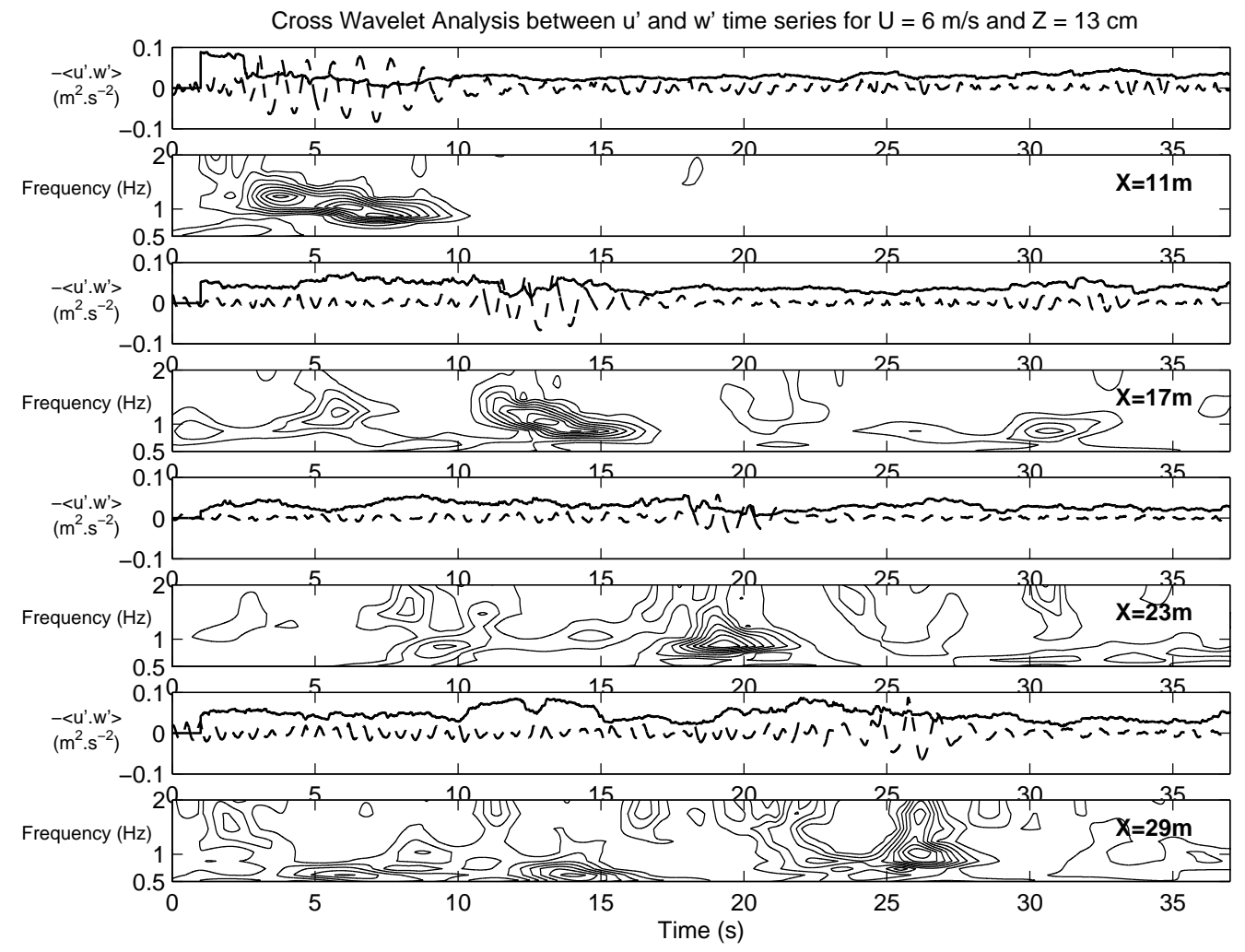

FIgURE 12. Cross-wavelet power for wind velocity fluctuations $u^{\prime}$ and $w^{\prime}$ at height $z=13 \mathrm{~cm}$, for mean wind velocity $U=6 \mathrm{~m} / \mathrm{s}$. The vertical and bottom axes are the frequency and time respectively.

which has been observed by Clamond et al (2006) when extreme waves are due to modulational phenomenon or envelope-soliton collision, can be extended to extreme waves due to spatio-temporal focusing. Furthermore, the figure emphasizes two main features pointed out previously: The downwind shift of the focus point and time duration of the extreme wave event increase with wind velocity. Notice that the frequency minimum decreases as wind velocity increases. The curves exhibit a minimum which corresponds to a maximum of the group velocity calculated from the linear dispersion relation. The maxima of the associated group velocity are $0.814 \mathrm{~m} / \mathrm{s}, 0.819 \mathrm{~m} / \mathrm{s}, 0.825 \mathrm{~m} / \mathrm{s}$ and $0.841 \mathrm{~m} / \mathrm{s}$ for $U=0 \mathrm{~m} / \mathrm{s}, 4 \mathrm{~m} / \mathrm{s}, 6 \mathrm{~m} / \mathrm{s}$ and $8 \mathrm{~m} / \mathrm{s}$ respectively. These values of the group velocity are less than those of the velocity calculated previously. The deviations can be explained by nonlinear effects. Indeed, the extreme waves are strongly nonlinear and their envelope velocities on average are larger than the group velocities calculated from the linear dispersion relation. Nevertheless, as emphasized previously the group velocity fluctuates during extreme wave event and may be locally less than the linear value. This feature has been pointed out by ? in the case of nonlinear spatio-temporal focusing due to BenjaminFeir instability. This tendency which is also observed experimentally for the dispersive focusing investigated herein as been confirmed by numerical simulations.

Figure 20 shows the wind stress as a function of $z$ for $U=4 \mathrm{~m} / \mathrm{s}$ with or without the presence of focusing wave groups. It can be seen that when there is no extreme wave event the wind stress varies of $20 \%$ from $z=10 \mathrm{~cm}$ to $z=19 \mathrm{~cm}$ while it varies of $130 \%$ 
Cross Wavelet Analysis between $p^{\prime}$ and $\eta_{x}$ time series for $U=6 \mathrm{~m} / \mathrm{s}$ and $Z=13 \mathrm{~cm}$
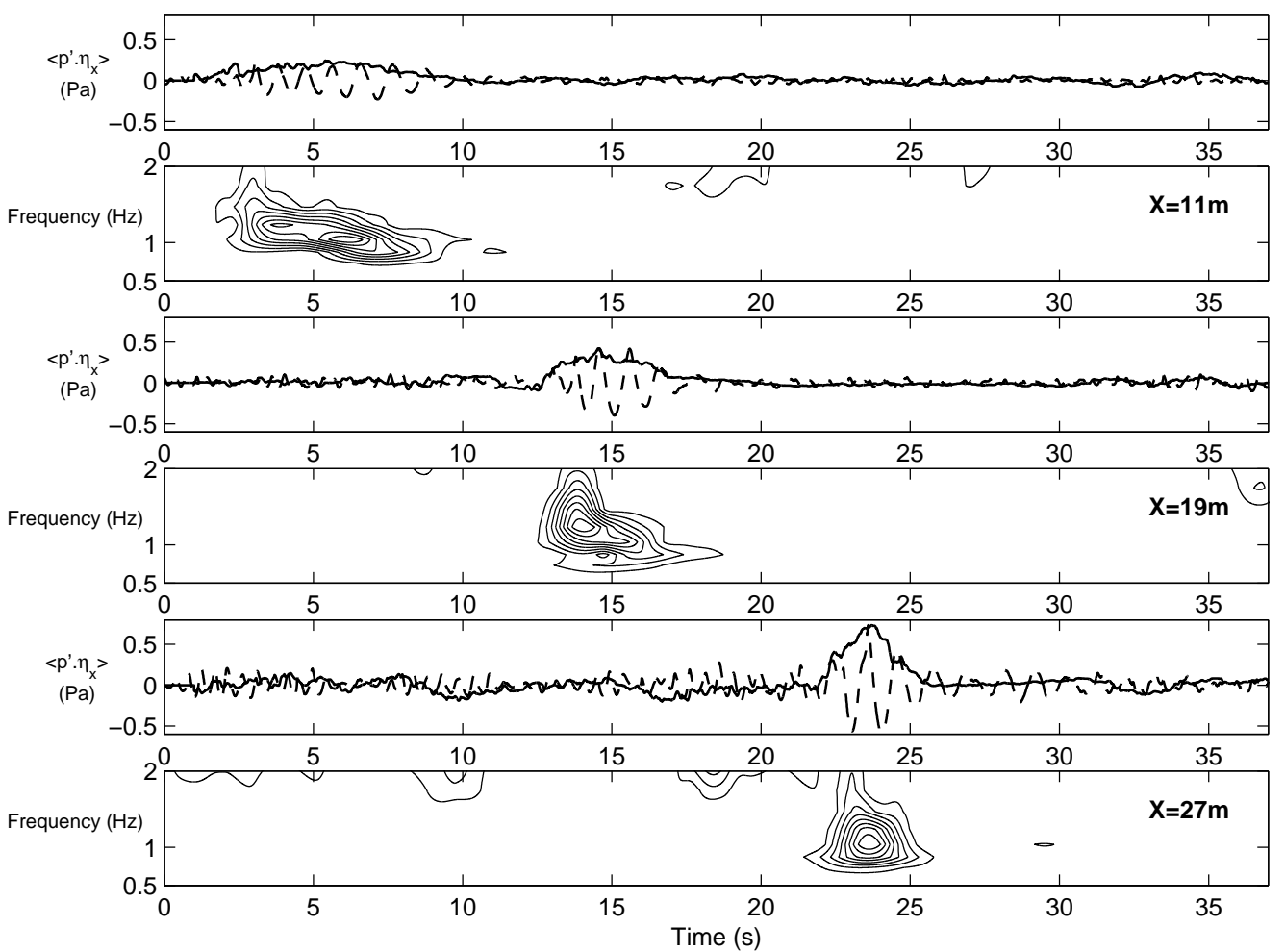

Figure 13. Cross-wavelet power for pressure fluctuations $p^{\prime}$ and $\partial \eta / \partial x$ at height $z=13 \mathrm{~cm}$, for mean wind velocity $U=6 \mathrm{~m} / \mathrm{s}$. The vertical and bottom axes are the frequency and time respectively.

between the same values of the altitude when extreme wave events occur. This feature can be explain by a strong longitudinal mean pressure gradient due to the modification of the air flow structure in presence of extreme wave events or strongly modulated wave trains.

The previous experimental results suggest that air flow separation could explain the strong increase of the transfer of momentum and energy during extreme wave events. To verify the validity of this assumption a series of experiments using an original probe developed at the laboratory by ? to detect air flow separation mechanism (AFS) have been conducted. The AFS probe is presented in Figure 21. A hot wire and a cold wire separated each other from $1 \mathrm{~mm}$ in the direction of the mean wind direction are installed on a wavefollower. Any temperature fluctuation can be detected by the cold wire when it is located in the hot wake generated by the hot wire. In presence of air flow separation, a reverse flow directed towards the upstream direction can occur in the vicinity of the leeward face of the crest (N. Reul \& Giovanangeli (1999)) which produces both a positive temperature fluctuation measured by the cold wire and a negative wind velocity fluctuation measured by the hot wire. Using the wave-follower, the AFS probe has been located in the close vicinity of the instantaneous water wave surface and particularly close to the wave trough. Figure 22 that corresponds to the case $U=4 \mathrm{~m} / \mathrm{s}$ shows the records of the elevation of the interface, elevation of the AFS probe fixed at $3 \mathrm{~cm}$ from the water wave surface, output voltage given by the cold and hot wires. In this figure one can observe that during the 


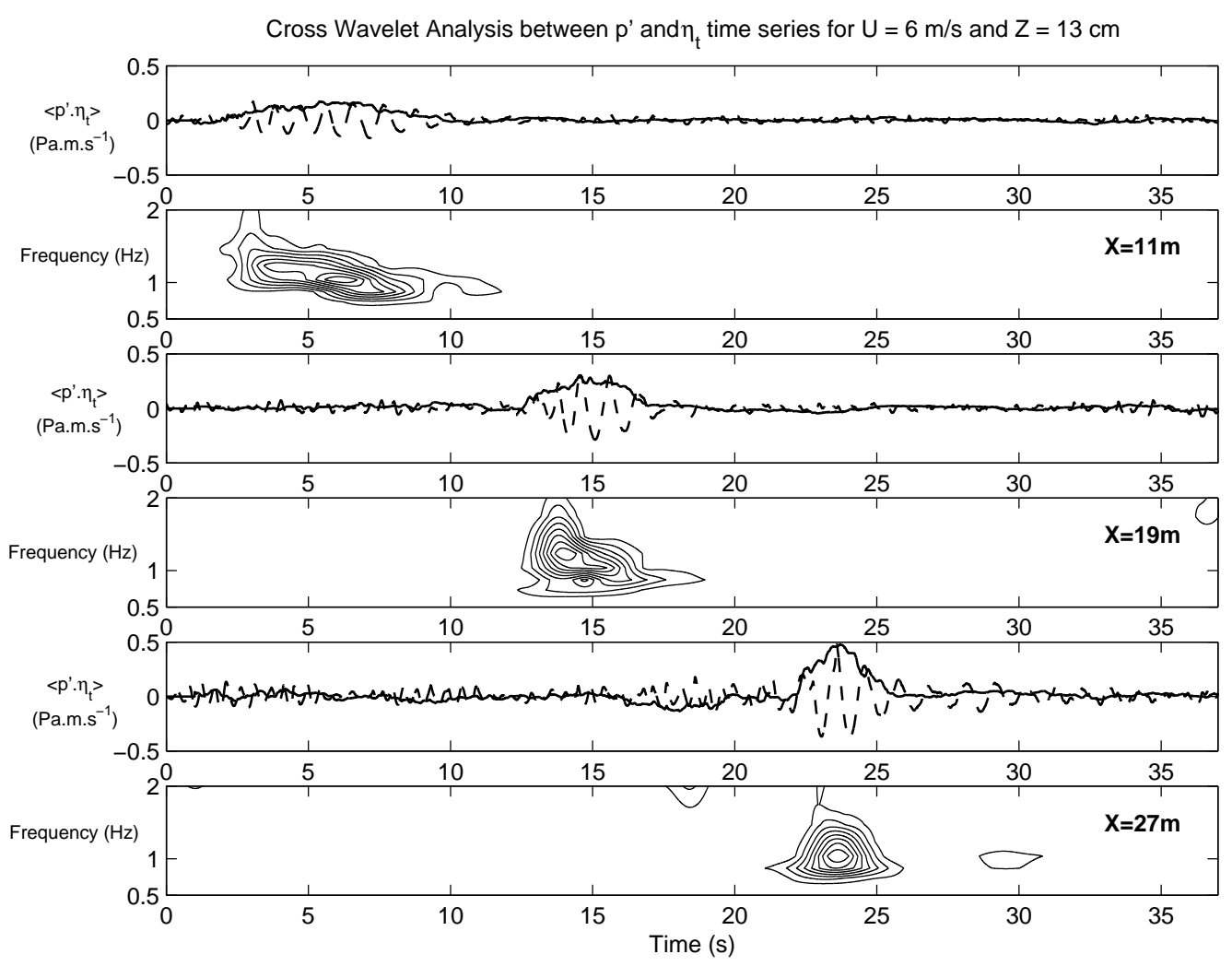

Figure 14. Cross-wavelet power for pressure fluctuations $p^{\prime}$ and $\partial \eta / \partial t$ at height $z=13 \mathrm{~cm}$, for mean wind velocity $U=6 \mathrm{~m} / \mathrm{s}$. The vertical and bottom axes are the frequency and time respectively.

occurrence of the burst of the local wave slope appears a decrease of the wind velocity and a positive temperature fluctuation measured by the cold wire. Hence, even for a wind velocity of $4 \mathrm{~m} / \mathrm{s}$ an air flow separation occurs when the local wave slope of the interface reaches a threshold value which as been evaluated herein close to 0.35 . This suggests that the local wave slope is a significant parameter which is highly correlated to the air flow separation phenomenon. In presence of steep wave events, the wave age is not the unique parameter to be considered, the local wave slope is a significant parameter too. Furthermore, it has been observed that the occurrence of air flow separation is generally accompanied by breaking. This is in agreement with the results of Banner \& Melville (1976).

In section 3 , the critical slopes that will be used in the numerical simulations of the spatio-temporal focusing are chosen close to the experimental threshold 0.35.

\section{Numerical simulations}

One of the main objectives of the present section is to study frequency modulated wave trains generated in a numerical wave tank to compare their behaviour with experiments with and without wind. To consider conditions similar to those of the previous experiments we used a numerical wave tank based on a boundary integral equation method (BIEM). In the previous experiments sporadic breaking have been observed. To avoid 
$X(T)$ of Maximum Wavelet Analysis of $\eta$

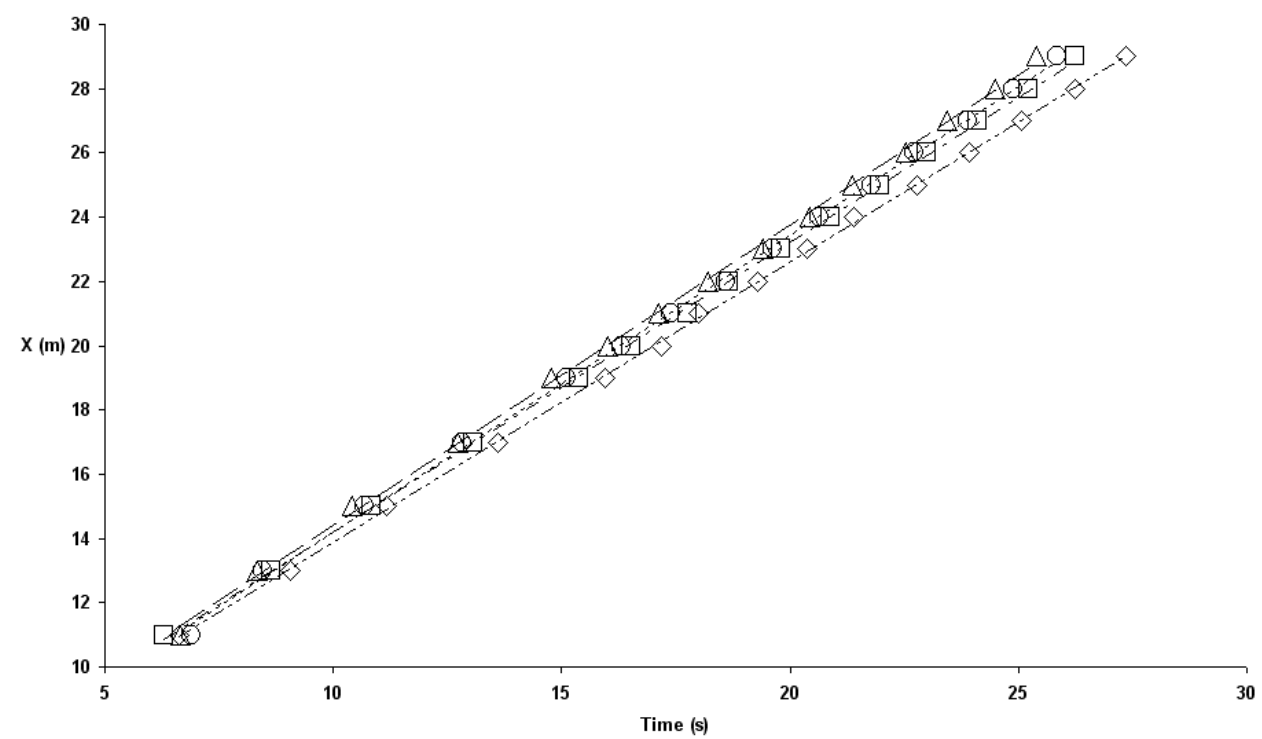

FiguRE 15. Trajectories of the maximum of the wavelet power spectrum of the surface elevation for several values of the wind velocity in the $x-t$ plane. $U=0 \mathrm{~m} / \mathrm{s}(\diamond), U=4 \mathrm{~m} / \mathrm{s}(\square), U=6 \mathrm{~m} / \mathrm{s}$ $(\bigcirc), U=8 \mathrm{~m} / \mathrm{s}(\triangle)$

\section{$X(T)$ of Maximum Cross Wavelet Analysis between $\mathrm{u}^{\prime}$ and $\mathrm{w}^{\prime}$ for $\mathrm{U}=6 \mathrm{~m} / \mathrm{s}$}

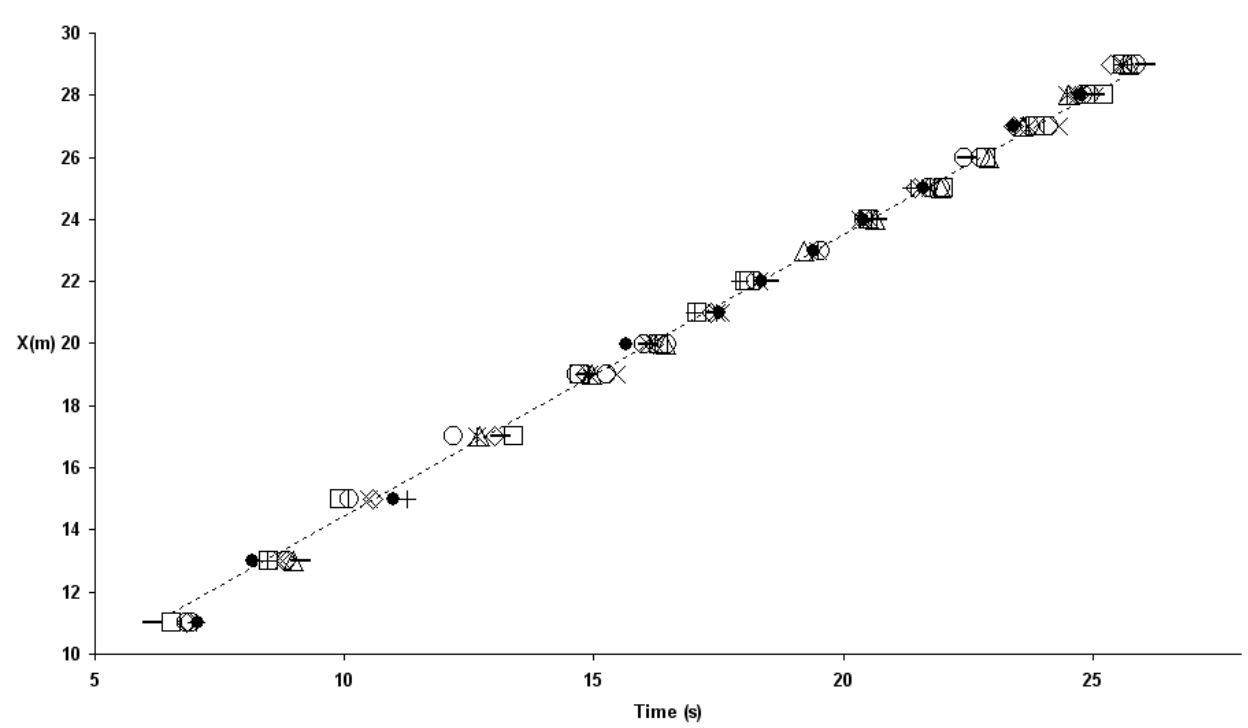

Figure 16. Trajectories of the maximum of the cross-wavelet power for $u^{\prime}$ and $w^{\prime}$ at several altitudes above the mean water level for $U=6 \mathrm{~m} / \mathrm{s}$ in the $x-t$ plane. $Z=13 \mathrm{~cm}(\bullet), Z=14 \mathrm{~cm}$ (口), $Z=15 \mathrm{~cm}(\circ), Z=16 \mathrm{~cm}(\times), Z=17 \mathrm{~cm}(*), Z=18 \mathrm{~cm}(\bigcirc), Z=19 \mathrm{~cm}(+), Z=20 \mathrm{~cm}$ $(\triangle), Z=25 \mathrm{~cm}(-), Z=30 \mathrm{~cm}(\diamond)$ 
$X(T)$ of Maximum Cross Wavelet Analysis between $u^{\prime}$ and $w^{\prime}$ for $Z=14 \mathrm{~cm}$

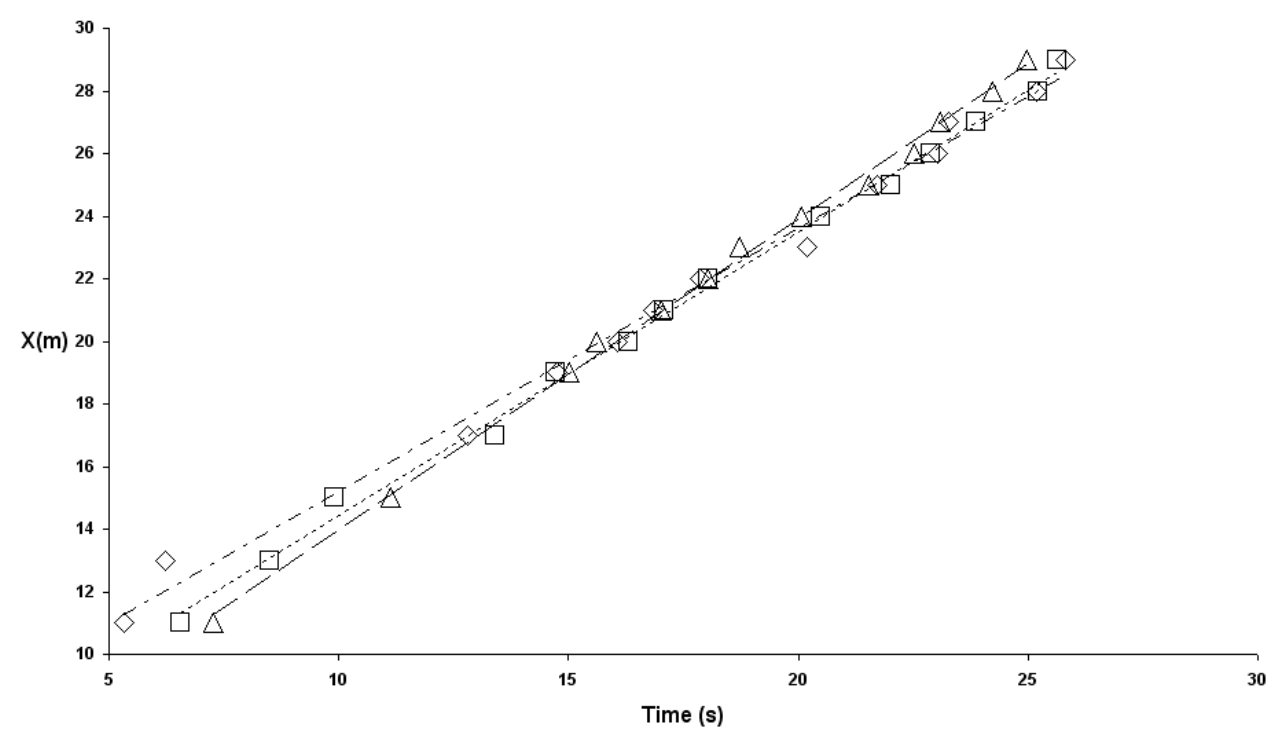

FiguRE 17. Trajectories of the maximum of the cross-wavelet power for $u^{\prime}$ and $w^{\prime}$ at altitude $z=14 \mathrm{~cm}$, for several wind velocities in the $x-t$ plane. $U=4 \mathrm{~m} / \mathrm{s}(\diamond), U=6 \mathrm{~m} / \mathrm{s}(\square), U=8 \mathrm{~m} / \mathrm{s}$ $(\triangle)$

\section{$X(T)$ of Maximum Cross Wavelet Analysis between $\mathrm{p}^{\prime}$ and $\partial \eta / \partial \mathrm{t}$ for $\mathrm{U}=6 \mathrm{~m} / \mathrm{s}$}

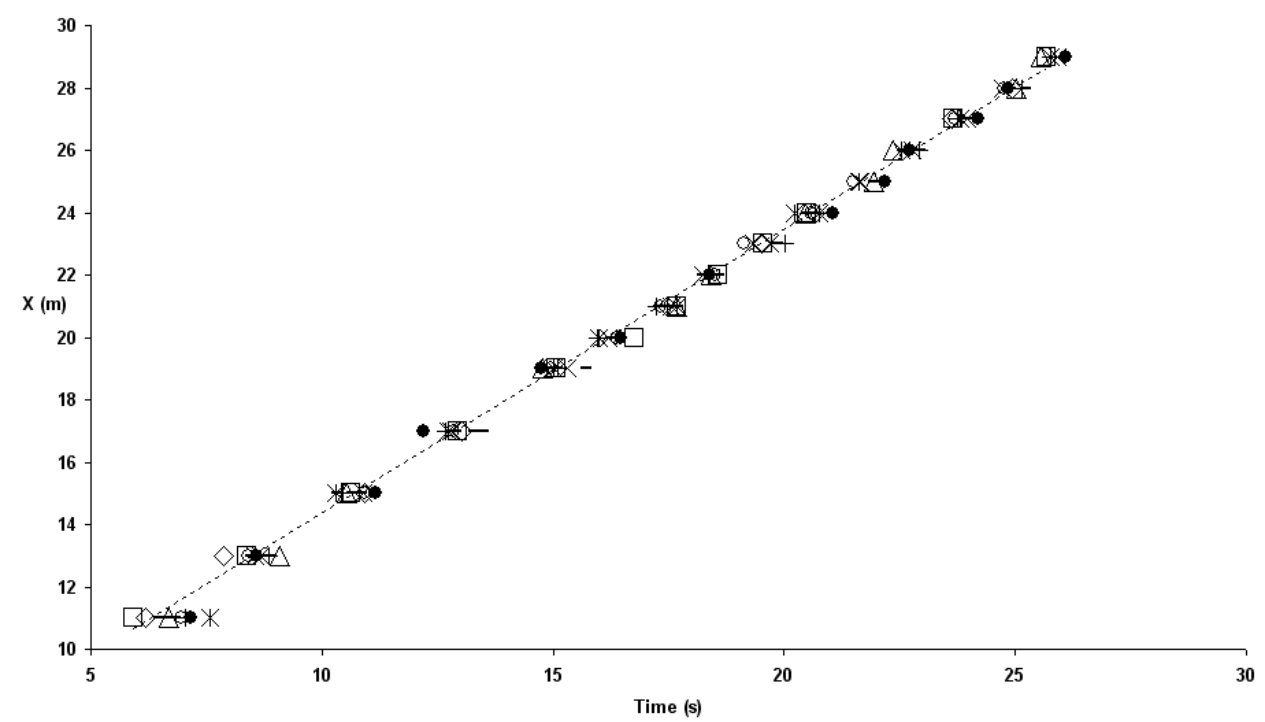

FiguRE 18. Trajectories of the maximum of the cross-wavelet power for $p^{\prime}$ and $\partial \eta / \partial t$ at several altitudes above the mean water level for $U=6 \mathrm{~m} / \mathrm{s}$, in the $x-t$ plane. $Z=13 \mathrm{~cm}(\diamond), Z=14 \mathrm{~cm}$ $(\square), Z=15 \mathrm{~cm}(\triangle), Z=16 \mathrm{~cm}(\times), Z=17 \mathrm{~cm}(*), Z=18 \mathrm{~cm}(\bullet), Z=19 \mathrm{~cm}(+), Z=20 \mathrm{~cm}$ $(-), Z=25 \mathrm{~cm}(-), Z=30 \mathrm{~cm}(\bigcirc)$ 


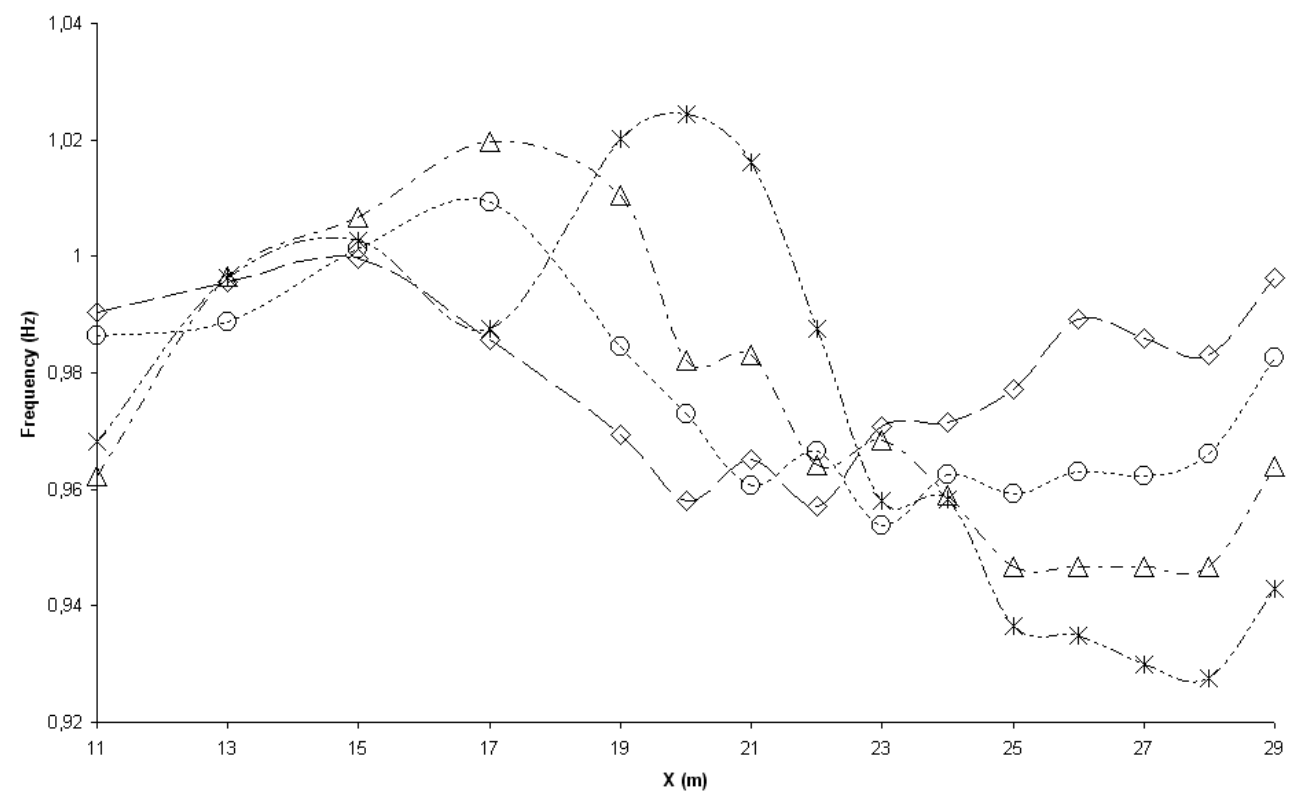

FiguRE 19. Evolution of the frequency of the maximum of the wavelet power spectrum of the surface elevation with the fetch for several values of the wind velocity. $U=0 \mathrm{~m} / \mathrm{s}(\diamond), U=4 \mathrm{~m} / \mathrm{s}$ $(\bigcirc), U=6 \mathrm{~m} / \mathrm{s}(\triangle), U=8 \mathrm{~m} / \mathrm{s}(*)$

this two-phase dissipative process that our numerical model cannot simulate, a third series of experiments has been conducted to compare both experimental and numerical results and also to check the validity of the numerical wave tank.

Beside the focusing due to dispersion of a chirped wave group, another mechanism, the modulational instability or Benjamin-Feir instability of uniform wave trains, can generate extreme wave events. This instability was discovered by Benjamin \& Feir (1967). At the same time Zakharov (1968) using a Hamiltonian formulation of the water wave problem arrived to same instability. The nonlinear evolution of this periodic phenomenon is investigated numerically using a high-order spectral method (HOSM), without experimental counterpart. The question is to know how evolve extreme wave events due to modulational instability under wind action. How are modified the amplification and time duration of these waves under wind effect? Are these effects similar or different from those observed in the case of extreme wave events due to the spatio-temporal focusing discussed previously?

\subsection{Wind modelling: The modified Jeffreys' sheltering theory}

In section 2 it was demonstrated experimentally for a wind velocity $U=4 \mathrm{~m} / \mathrm{s}$ that steep wave events occurring in water wave groups are accompanied by air flow separation. Furthermore, a careful inspection of Figure 5 suggests that a significant wind effect takes place when the steep wave event occurs. The focusing stage is almost independent of the wind velocity. Deviations can be observed only in the vicinity of the focus point where the waves become steep. This observation reinforces the idea that separation of the air flow in the lee of the wave crests is responsible for the growth and persistence of steep waves. The Jeffreys' sheltering mechanism which was introduced by Jeffreys (1925) could be used as wind modelling. Since air flow separation occurs only over steep waves, the Jeffreys' sheltering mechanism has to be applied locally in time and space and not 
Evolution of $<-$ u'. $^{\prime} w^{\prime}>$ as function of $Z$ for $U=4 \mathrm{~m} / \mathrm{s}$

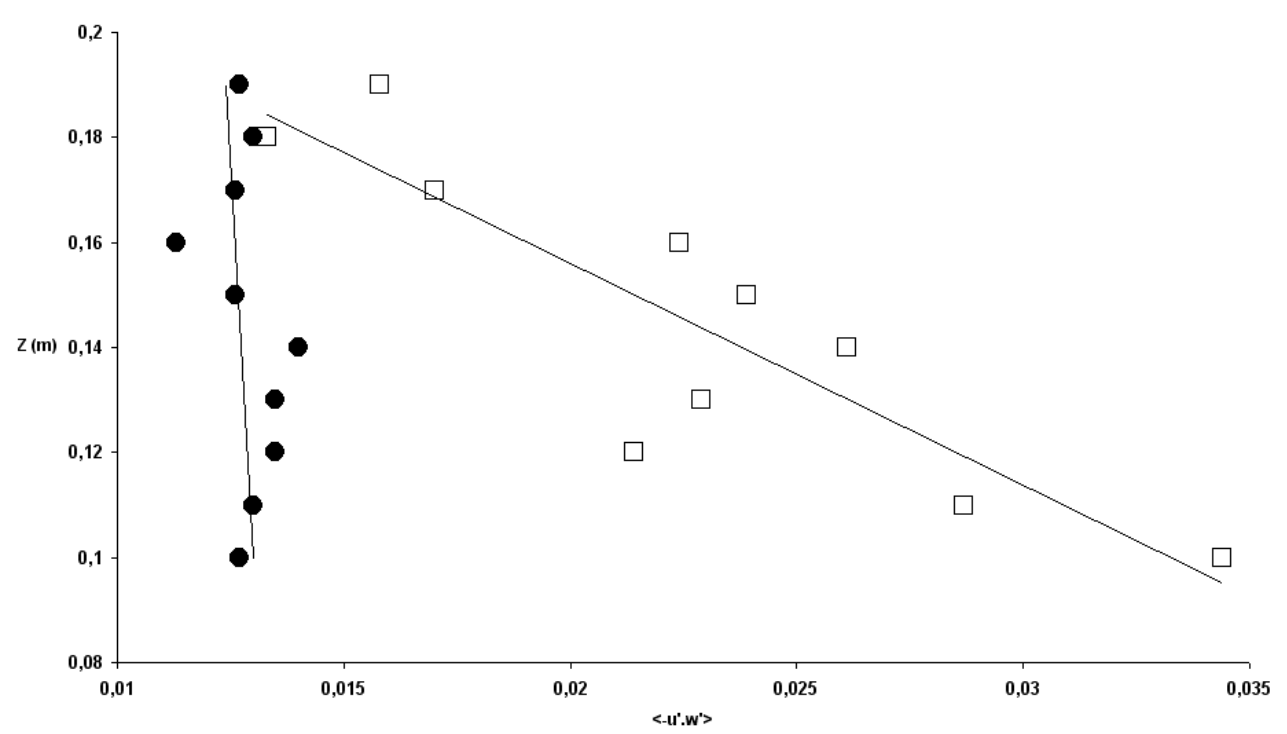

Figure 20. The wind stress $-<u^{\prime} w^{\prime}>$ as a function of the altitude $z$ above the mean surface elevation for $U=4 \mathrm{~m} / \mathrm{s}$ : $(\square)$ over the focusing group and $(\bullet)$ without focusing group.

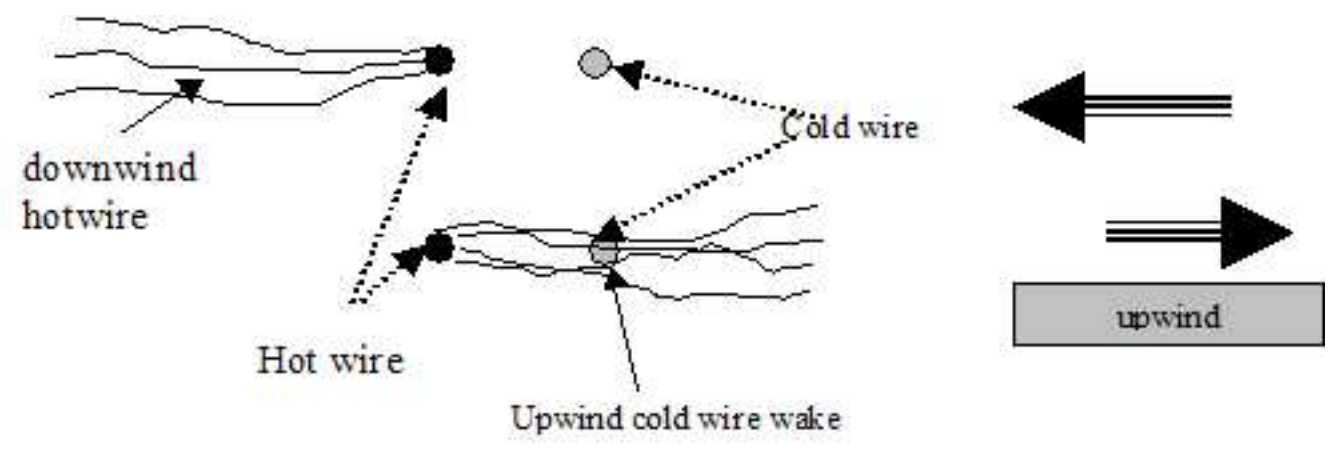

FIGURE 21. Schematic representation of the air flow separation probe

permanently over the whole wave field. It is well known that this mechanism cannot be applied continuously over water waves. This mechanism is working only when air flow separation occurs over steep waves (Banner \& Melville (1976); Kawai (1982)).

Previous works on rogue wave have not considered the direct effect of wind on their dynamics. It was assumed that they occur independently of wind action, that is, far away from storm areas where wind wave fields are formed. Herein the Jeffreys' theory (see Jeffreys (1925)) is invoked for the modelling of the pressure, $p_{a}$. Jeffreys proposed a plausible mechanism to explain the phase shift of the atmospheric pressure, $p_{a}$, needed for an energy transfer from wind to the water waves. He suggested that the energy transfer was due to the form drag associated with the flow separation occurring on the leeward side of the crests. The air flow separation would cause a pressure asymmetry with respect to the wave crest resulting in a wave growth. This mechanism can be invoked only if the waves are sufficiently steep to produce air flow separation. Banner \& Melville (1976) have shown that separation occurs over breaking waves. For weak or moderate steepness of the 

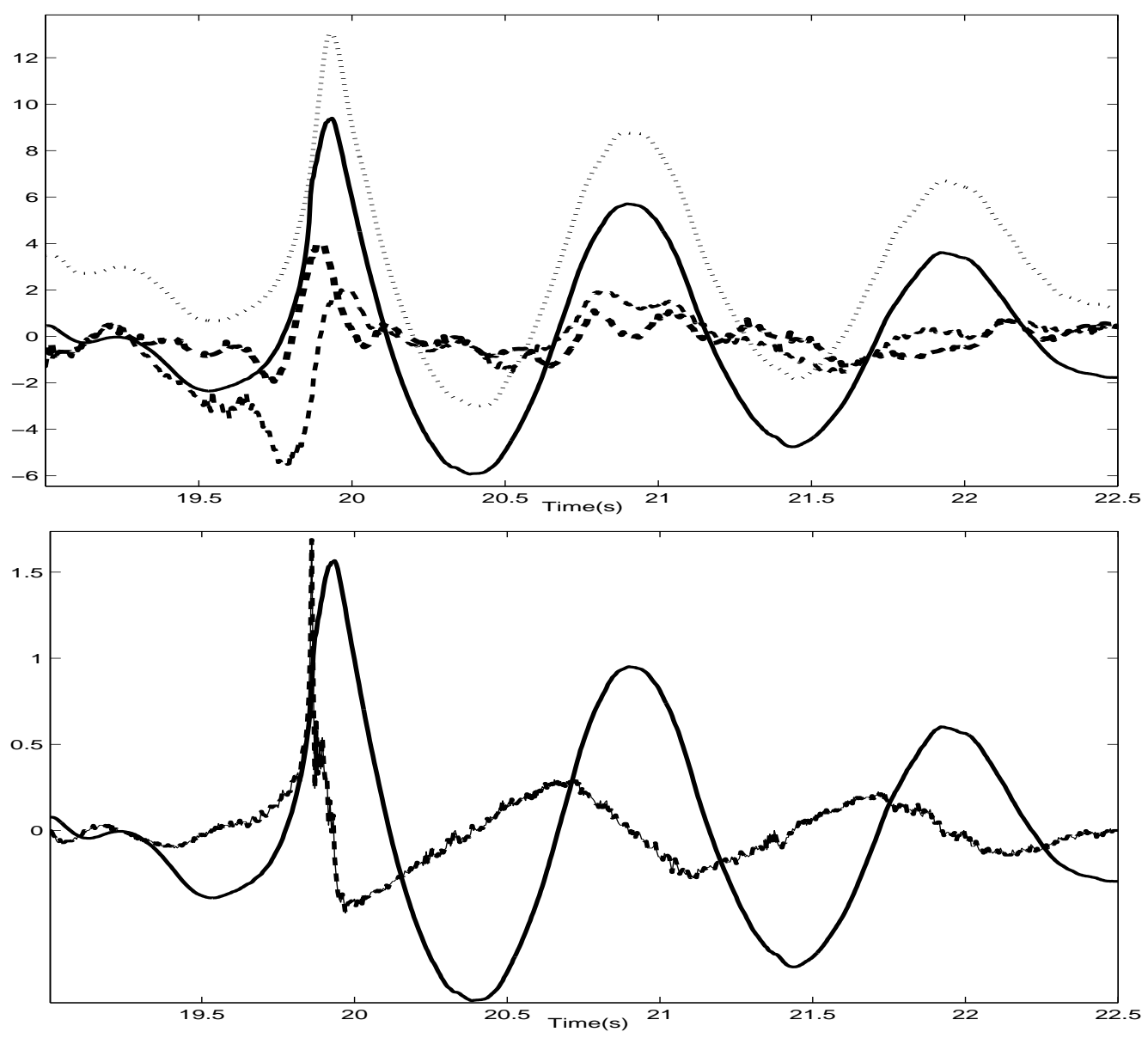

FIGURE 22. Wave surface elevation (solid line), AFS probe elevation (dotted line), cold wire ouput (thick dashed line) and hot wire output (thin dashed line) as a function of time for $U=4 \mathrm{~m} / \mathrm{s}$ (top). Local longitudinal wave slope (dashed line) and wave surface elevation as a function of time for $U=4 \mathrm{~m} / \mathrm{s}$ (bottom).

waves this phenomenon cannot apply and the Jeffreys' sheltering mechanism becomes irrelevant.

Following Jeffreys (1925), the pressure at the interface $z=\eta(x, t)$ is related to the local wave slope according to the following expression

$$
p_{a}=\rho_{a} s(U-c)^{2} \frac{\partial \eta}{\partial x} .
$$

where the constant, $s$ is termed the sheltering coefficient, $U$ is the wind speed, $c$ is the wave phase velocity and $\rho_{a}$ is atmospheric density. The sheltering coefficient, $s=0.5$, has been calculated from experimental data. In order to apply the relation (3.1) for only steep waves we introduce a threshold value for the slope $(\partial \eta / \partial x)_{c}$. When the local slope of the waves becomes larger than this critical value, the pressure is given by equation (3.1) otherwise the pressure at the interface is taken equal to a constant which is chosen equal to zero without loss of generality. This means that wind forcing is applied locally in time and space. According to the experiments, the critical value of the slope, $(\partial \eta / \partial x)_{c}$, is chosen close to 0.35 , in the range $(0.30-0.40)$ for the spatio-temporal focusing. For the nonlinear 


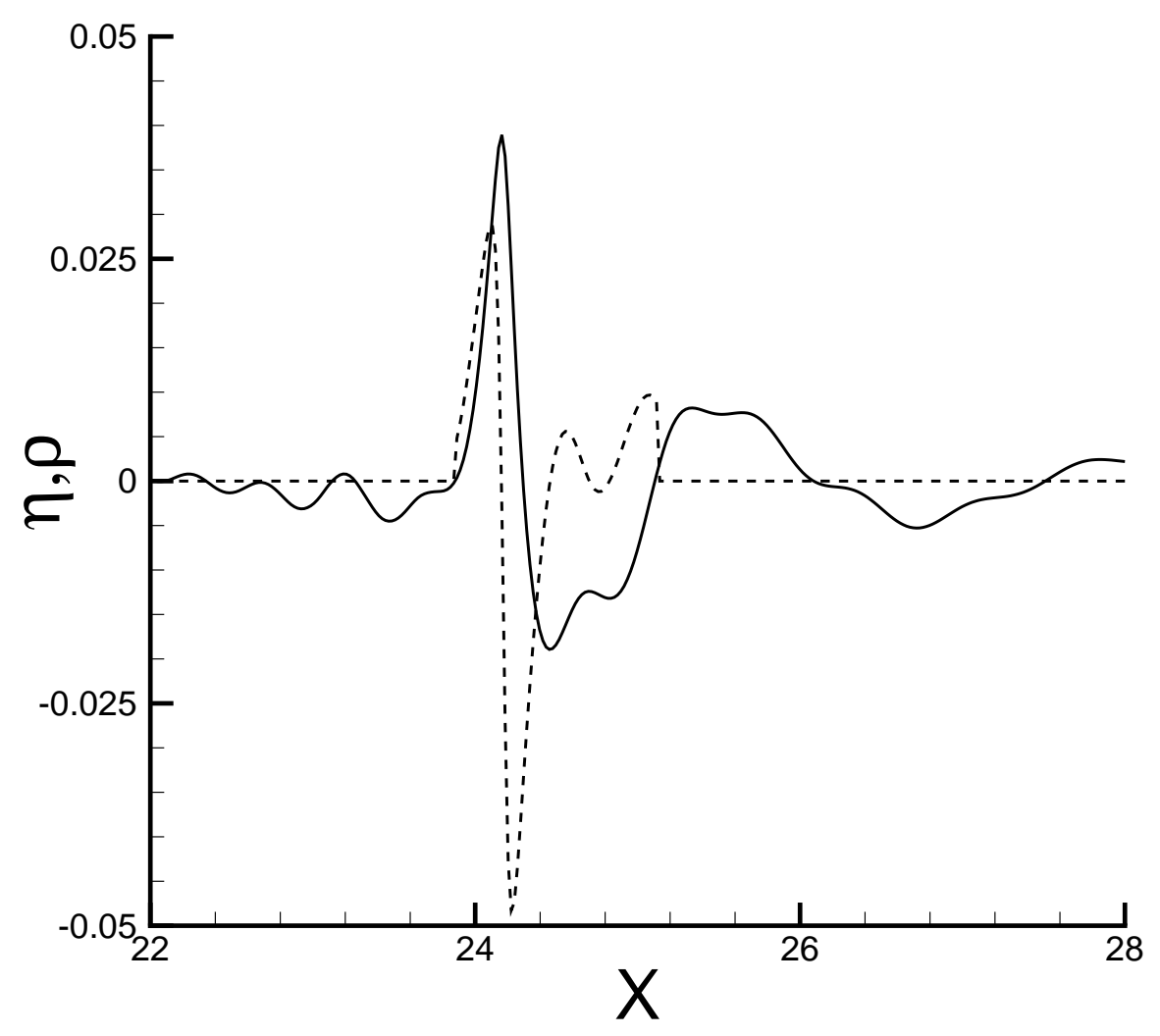

FiguRE 23. Pressure at the interface given in $10^{-1} \mathrm{HPa}$ (dashed line) and surface elevation given in $m$ (solid line) as a function of $x$.

focusing due to modulational instability we used higher values to avoid a rapid evolution towards breaking. When the critical value is low, the transfer of energy from the wind to the waves yields to wave breaking and when it is too high this transfer becomes negligible to influence the wave dynamics. The choice of the value of the sheltering coefficient is also of importance. This coefficient has been computed experimentally. We have not perform a systematic study on the influence of $(\partial \eta / \partial x)_{c}$ and $s$ on the wind-wave coupling. Our main purpose is to show that the application of the modified Jeffreys mechanism could explain simply some features of the interaction between wind and strongly modulated water wave groups.

Figure 23 shows the pressure distribution at the interface in the vicinity of the crest, given by equation (3.1), for a threshold value close to the slope corresponding to the Stokes' corner.

\subsection{Basic equations}

We consider two-dimensional propagating nonlinear gravity wave trains on the surface of an inviscid and incompressible fluid. Under the assumption that the motion is irrotational, the governing equations are the Laplace equation and nonlinear boundary 
conditions

$$
\begin{gathered}
\Delta \phi=0, \quad \text { for } \quad z<\eta(x, t), \\
\lim _{z \rightarrow-\infty} \nabla \phi=0 \\
\frac{\partial \eta}{\partial t}+\frac{\partial \eta}{\partial x} \frac{\partial \phi}{\partial x}-\frac{\partial \phi}{\partial z}=0, \quad z=\eta(x, t), \\
\frac{\partial \phi}{\partial t}+\frac{1}{2} \nabla \phi \cdot \nabla \phi+g \eta=-\frac{p_{a}}{\rho_{w}}, \quad z=\eta(x, t),
\end{gathered}
$$

where $\phi(x, z, t)$ is the velocity potential, $z=\eta(x, t)$ is the equation of the surface, $g$ is the acceleration due to gravity, $p_{a}$ is the atmospheric pressure, $x$ and $z$ are the horizontal and vertical coordinates respectively and $t$ is the time.

\subsection{The spatio-temporal focusing}

Herein we considered a numerical wave tank simulating the experimental water wave tank described in the previous section. The gravity wave train is generated by a pistontype wavemaker. An absorbing beach located at the end of the wave tank dissipates the incident wave energy.

The Laplace equation (3.2) is solved within a domain bounded by the water surface and solid boundaries of the numerical wave tank. The condition on the solid boundary writes

$$
\nabla \phi \cdot \boldsymbol{n}=\boldsymbol{v} \cdot \boldsymbol{n}, \quad \text { on } \quad \partial \Omega_{\mathrm{S}},
$$

where $\partial \Omega_{\mathrm{S}}$ corresponds to solid boundaries, $\boldsymbol{v}$ is the velocity of the solid boundaries, set equal to zero on the horizontal bottom and downstream wall of the wave tank and equal to the velocity of the piston at any point of the wavemaker, and $\boldsymbol{n}$ is the unit normal vector to the boundaries.

A Lagrangian description of the water surface is used

$$
\begin{aligned}
& \frac{D \eta}{D t}=\frac{\partial \phi}{\partial z}, \\
& \frac{D x}{D t}=\frac{\partial \phi}{\partial x},
\end{aligned}
$$

where $x$ is the abscissa of the water surface and $D / D t=\partial / \partial t+\nabla \phi \cdot \nabla$.

Equation (3.7) is an alternative form of equation (3.4). The kinematic boundary condition writes as well

$$
\frac{D S}{D t}=0 .
$$

where $S(x, z, t)=\eta(x, t)-z=0$ is the water surface equation.

The dynamic boundary condition (3.5) is rewritten as follows

$$
\frac{D \phi}{D t}=\frac{1}{2}(\nabla \phi)^{2}-g \eta-\frac{p_{a}}{\rho_{w}},
$$

where the pressure, $p_{a}(x, t)$, at the water surface is given by equation (3.1), i.e. the Jeffreys' theory presented in section 4 is used for modelling wind effect on the extreme 
waves. Over waves presenting slopes less than a threshold value, the atmospheric pressure is uniform, set equal to zero without loss of generality.

The system of equations to solve is (3.2), (3.6), (3.7), (3.8), and (3.10). The method to integrate numerically this system is a boundary integral equation method (BIEM) with a mixed Euler-Lagrange (MEL) time marching scheme. The numerical method is based on the Green's second identity. For more details see the paper by Touboul et al. (2006).

A focusing wave train is generated by the piston wave maker, leading during the focusing stage to the generation of a extreme wave followed by a defocusing stage. The water surface and the solid boundaries (downstream wall, bottom and wave maker) are discretised by 2000 and 1000 meshes respectively, uniformly distributed. The time integration is performed using a RK4 scheme, with a constant time step of $0.01 \mathrm{~s}$. To avoid numerical instability the grid spacing $\Delta x$ and time increment $\Delta t$ have been chosen to the satisfy the following Courant criterion derived from the linearized surface conditions

$$
(\Delta t)^{2} \leqslant \frac{8 \Delta x}{\pi g}
$$

Figure 24 displays the experimental and computed surface elevation $\eta(t)$ at fetch $x=1 m$ while Figure 25 shows the surface elevation at several fetches, measured experimentally and computed numerically. The origin of the surface elevation corresponding to fetches $x=18 \mathrm{~m}$ and $x=21 \mathrm{~m}$ are located at 0.05 and 0.1 respectively. The data at fetch $x=1 m$ are in excellent agreement while discrepancies observed for steep waves at fetches $x=11 \mathrm{~m}, 18 \mathrm{~m}$ and $21 \mathrm{~m}$ are possibly due to local breaking. Nevertheless the phases of the numerical and experimental wave trains are the same demonstrating the efficiency of the numerical code to reproduce correctly the nonlinear evolution of water wave groups during the focusing-defocusing cycle.

In the first series of experiments described in section 2, spilling breaking events were observed, resulting in energy dissipation and in saturation in the growth of amplitude. The present model which is based on the assumption of inviscid fluid cannot describe energy dissipation. In our model, the transfer of energy from the wind to the water waves depends on the wind velocity and threshold wave slope value. If the latter value is low the energy transferred becomes high and breaking occurs.

To avoid breaking waves a third series of experiments and numerical simulations have been performed with an initial group of waves of weaker amplitude. For these experiments and simulations the period during which water waves are emitted is increased so that the initial group contains a greater number of waves. This explains why the amplification factor is greater for this case as it can be seen in Figure 26. The frequency of the wavemaker is varied linearly from $f_{\max }=1.85 \mathrm{~Hz}$ to $f_{\min }=0.8 \mathrm{~Hz}$ during $\Delta T=23.5 \mathrm{~s}$. The focusing mechanism is investigated with and without wind as well. A series of numerical simulations has been run for two values of the wind velocity: $U=0 \mathrm{~m} / \mathrm{s}$ and $6 \mathrm{~m} / \mathrm{s}$. Using equation (2.8), Figure 26 describes the spatial evolution of the amplification factor computed numerically. For $(\partial \eta / \partial x)_{c}=0.3$ a blow-up of the numerical simulation occurs due to the onset of breaking. This threshold value is too low and the transfer of energy from the wind to the steep waves yields to wave breaking. The threshold value of the slope beyond which the wind forcing is applied has been increased and is $(\partial \eta / \partial x)_{c}=0.4$. This value corresponds to a wave close to the limiting form for which the modified Jeffreys' theory applies. It can be observed that the numerical curves behave similarly to 


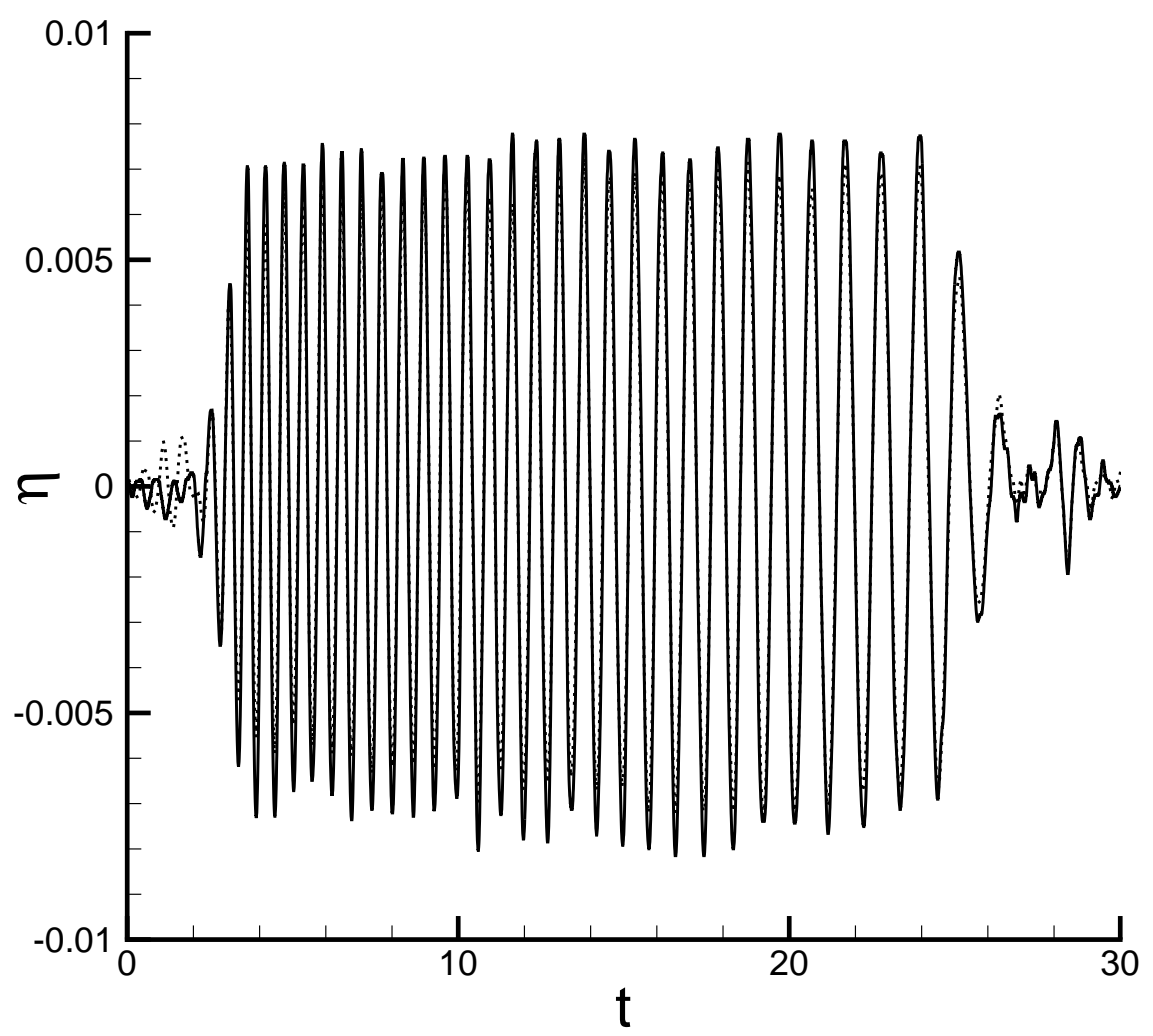

FiguRE 24. Surface elevation $(m)$ as a function of time $(s)$ at fetch $x=1 m$ : Experiments (solid line) and numerical simulation (dotted line) within the framework of the spatio-temporal focusing.

those plotted in Figure 5 and thus emphasize the asymmetry found in the experiments. The observed asymmetry between the focusing and defocusing regimes can be explained as follows. Without wind the amplitude of the extreme wave is decreasing during defocusing. In presence of wind, the modified Jeffreys' mechanism which is acting locally in time and space amplifies only the highest waves and hence delays their amplitude decrease during the very beginning of the defocusing stage. The competition between the dispersive nature of the water waves and the local transfer of energy from the wind to the extreme wave event leads to a balance of these effects at the maximum of modulation. This asymmetry results in an increase of the life time of the steep wave event which increases with the wind velocity. Hence the duration of the wind effect is relatively short to increase the amplification of the extreme wave event significantly. However a weak increase of the amplification factor is observed in presence of wind. The main effect of Jeffreys' sheltering mechanism is to sustain the coherence of the short group involving the steep wave event.

Figure 27 shows the experimental amplification factor and numerical amplification factor as a function of the normalized fetch $x / x_{\mathrm{f}}$ where $x_{\mathrm{f}}$ is the abscissa of the point of focus without wind. We can observe an excellent agreement between the experimental and nu- 


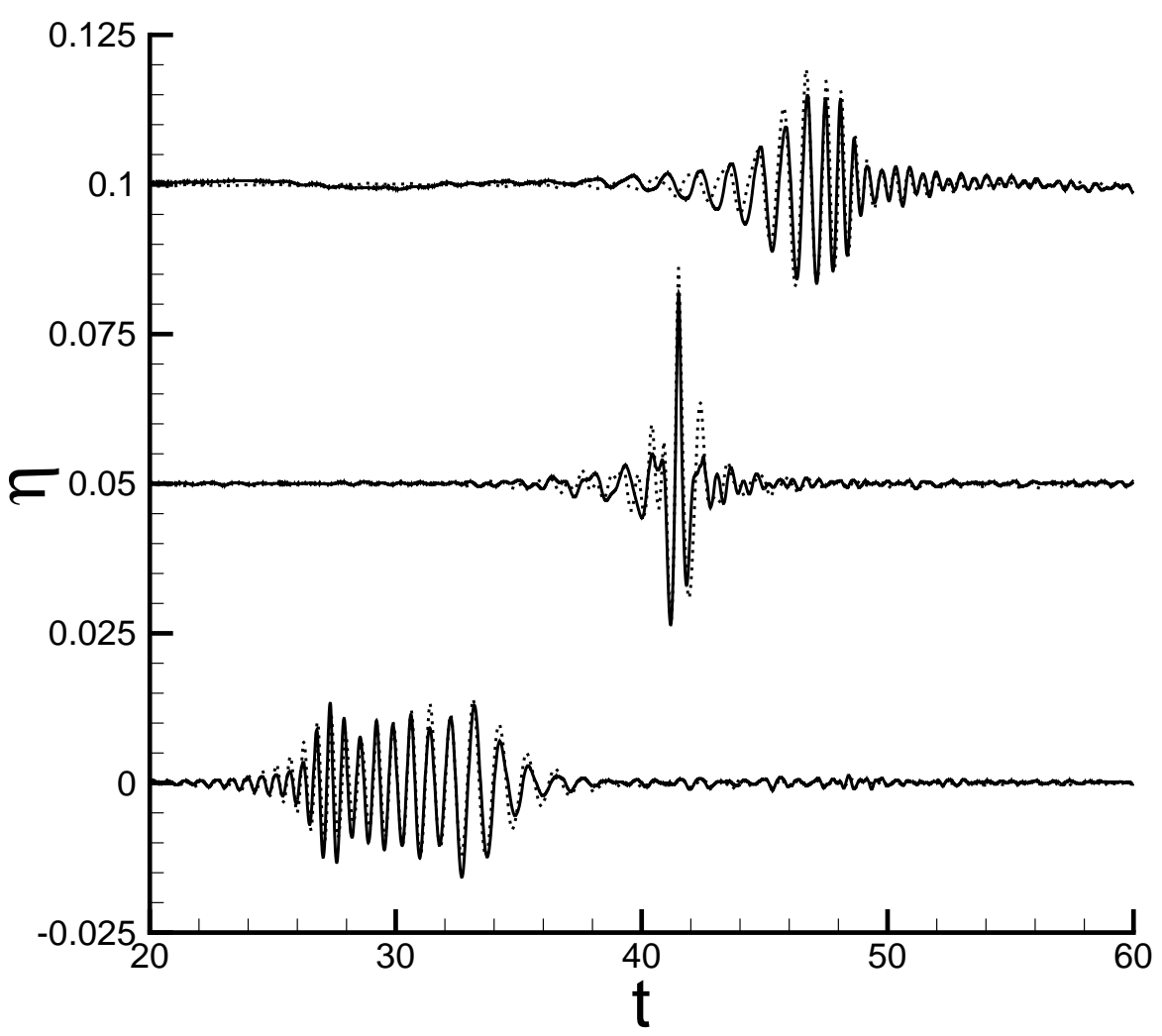

Figure 25. Surface elevation $(m)$ as a function of time $(s)$ at fetches $x=21 m$ (top), $x=18 m$ (middle) and $x=11 \mathrm{~m}$ (bottom): Experiments (solid line) and numerical simulation (dotted line) within the framework of the spatio-temporal focusing.

merical results. The experimental and numerical values of the abscissa of the focus point, $x_{\mathrm{f}}$, and amplification factor, $A$, are almost the same.

In presence of wind of velocity $U=6 \mathrm{~m} / \mathrm{s}$ Figure 28 (a) demonstrates that the numerical and experimental amplification factors disagree beyond the focus point. For the value $(\partial \eta / \partial x)_{c}=0.4$, the Jeffreys' sheltering mechanism is not effective enough in the present case while a reduction of the threshold value to 0.30 produces the onset of breaking at the focus point.

Wind waves are generally propagating in presence of current. Figure 28(b) corresponds to the spatio-temporal focusing in presence of wind and current with $(\partial \eta / \partial x)_{c}=0.3$. The wind velocity is $U=6 \mathrm{~m} / \mathrm{s}$ and a uniform following current corresponding to $2 \%$ of $U$ has been introduced to have the numerical value of the focus point equal to the experimental value. Generally, the current induced by wind is taken equal to $3 \%$ of the wind velocity. More information about the introduction of a current in the model can be found in the paper by Touboul et al. (2007) who considered the formation of rogue waves from transient wave trains propagating on current. The introduction of the following current prevents the onset of breaking. During extreme wave events the wind-driven current may play a significant role in the wind-wave interaction. The combined action of 


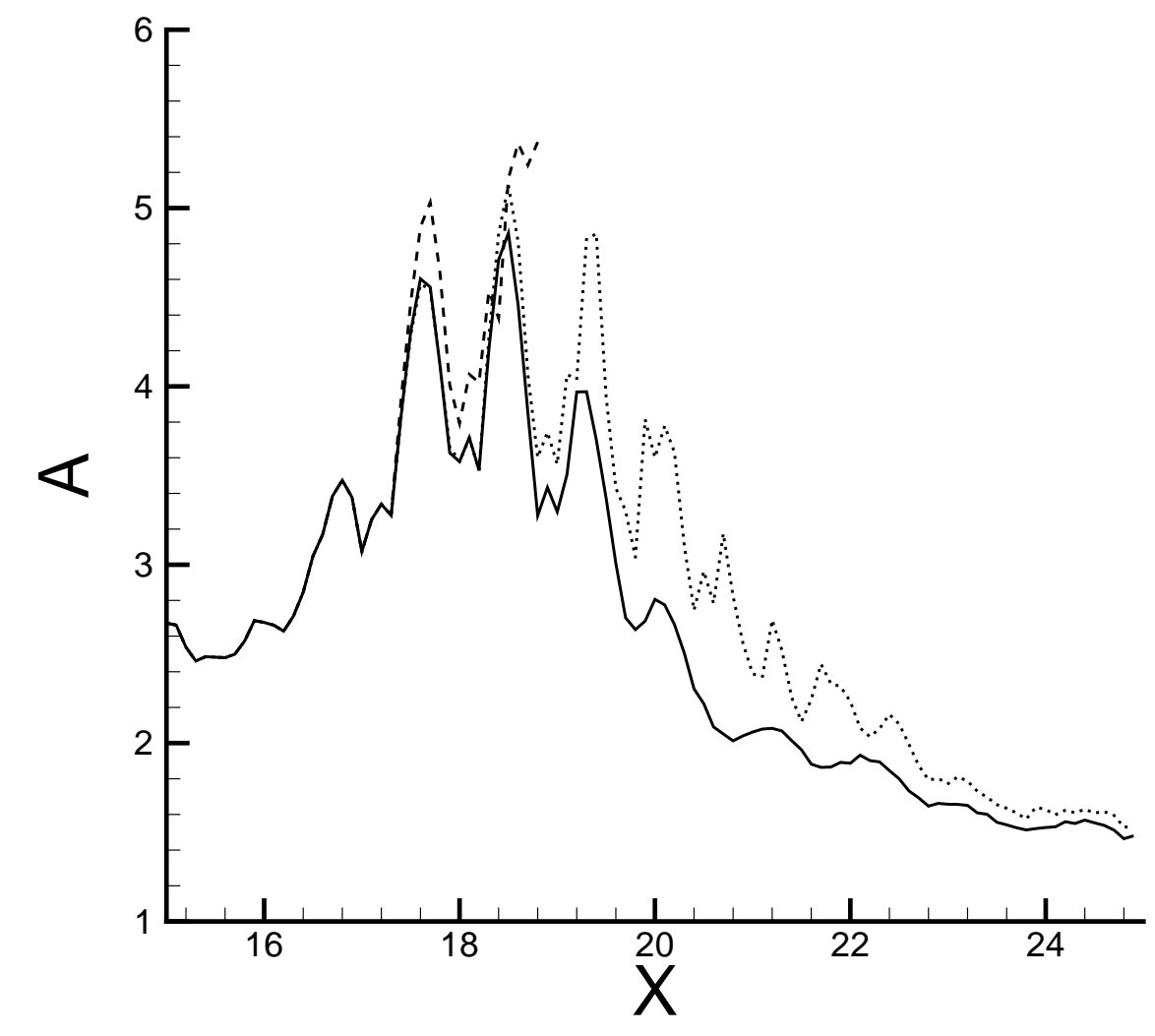

FIgURE 26. Numerical amplification factor $A(X, U)$ as a function of the distance (in $m$ ) for two values of the wind velocity within the framework of the spatio-temporal focusing: $U=0 \mathrm{~m} / \mathrm{s}$ (solid line), $U=6 \mathrm{~m} / \mathrm{s}$ and $(\partial \eta / \partial x)_{c}=0.4\left(\right.$ dotted line), $U=6 \mathrm{~m} / \mathrm{s}$ and $(\partial \eta / \partial x)_{c}=0.3($ dashed line)

the Jeffreys' sheltering mechanism and wind-driven current may sustain longer extreme wave events. We can observe a quite good agreement between the numerical simulation and experiment. The steep wave event is propagating over a longer distance (or period of time) in the numerical simulation and experiments as well.

To summarize we can claim that within the framework of the spatio-temporal focusing both experimental and numerical results are in qualitative good agreement even if some quantitative difference have been observed, namely when the wind-induced current is ignored. The importance of a following current on the evolution of the wave group has been emphasized as well.

\subsection{Focusing due to modulational instability}

Beside the focusing due to dispersion of a chirped wave group, another mechanism, the modulational instability or Benjamin-Feir instability (see the paper by Benjamin \& Feir (1967)) of uniform wave trains, can generate extreme wave events. This periodic phenomenon is investigated numerically using a high-order spectral method (HOSM) without experimental counterpart. The question is to know how evolve extreme wave events 


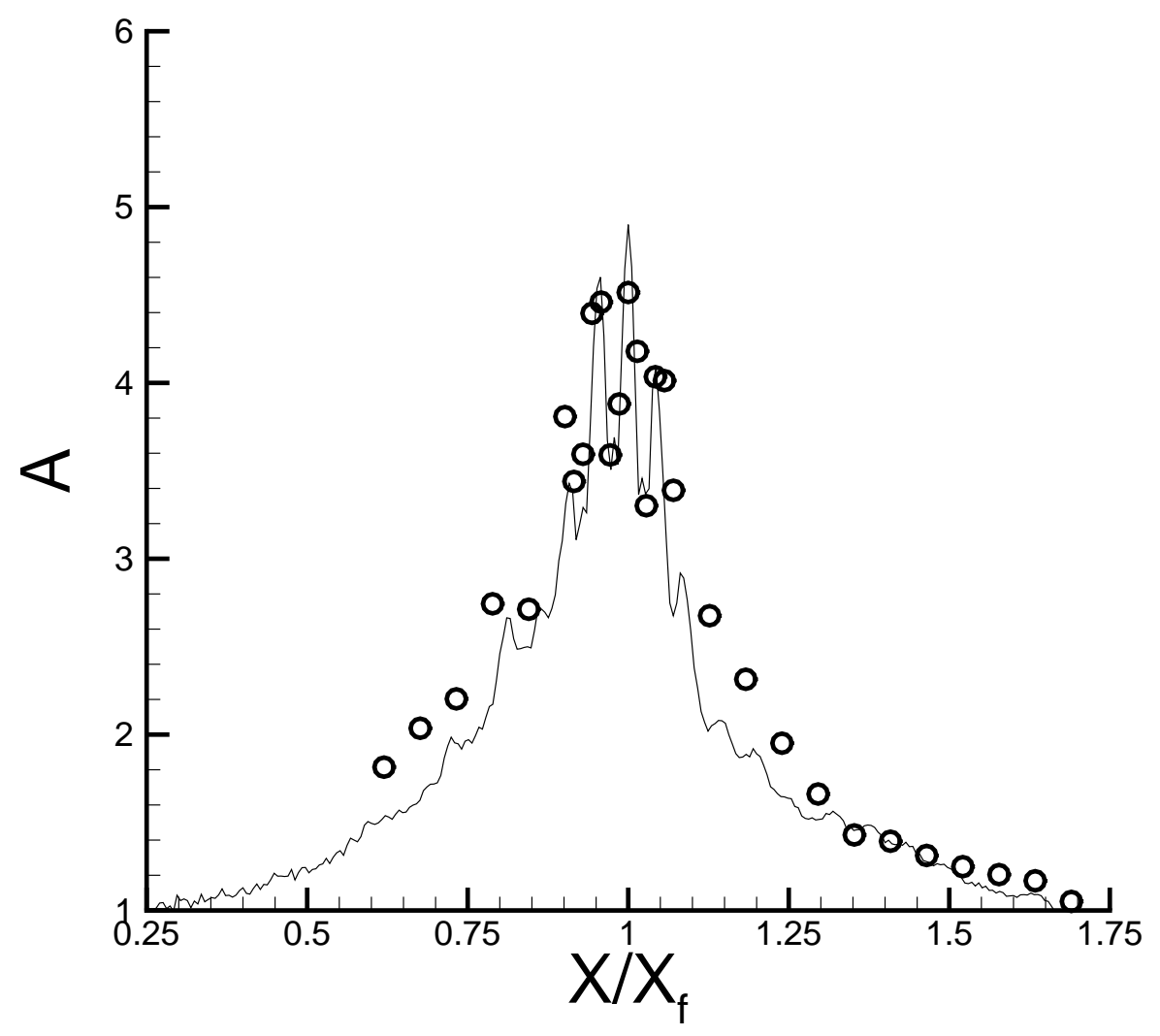

FiguRE 27. Numerical (solid line) and experimental (circle) amplification factor $A\left(X / X_{\mathrm{f}}, U\right)$ as a function of the normalized distance without wind within the framework of the spatio-temporal focusing

due to modulational instability under wind action. How are modified their amplification and time duration under wind effect? Are these effects similar or different from those observed in the case of extreme wave due to dispersive focusing?

Using the fully nonlinear equations Henderson et al. (1999) and Dyachenko \& Zakharov (2005) investigated numerically the onset of extreme wave events due to modulational instability but without considering wind influence.

Introducing the potential velocity at the free surface $\phi^{s}(x, t)=\phi(x, \eta(x, t), t)$, equations (3.4) and (3.5) writes

$$
\begin{gathered}
\frac{\partial \phi^{s}}{\partial t}=-\eta-\frac{1}{2} \nabla \phi^{s} \cdot \nabla \phi^{s}+\frac{1}{2} W^{2}\left[1+(\nabla \eta)^{2}\right]-p_{a} . \\
\frac{\partial \eta}{\partial t}=-\nabla \phi^{s} \cdot \nabla \eta+W\left[1+(\nabla \eta)^{2}\right] .
\end{gathered}
$$

where

$$
W=\frac{\partial \phi}{\partial z}(x, y, \eta(x, y, t), t) .
$$



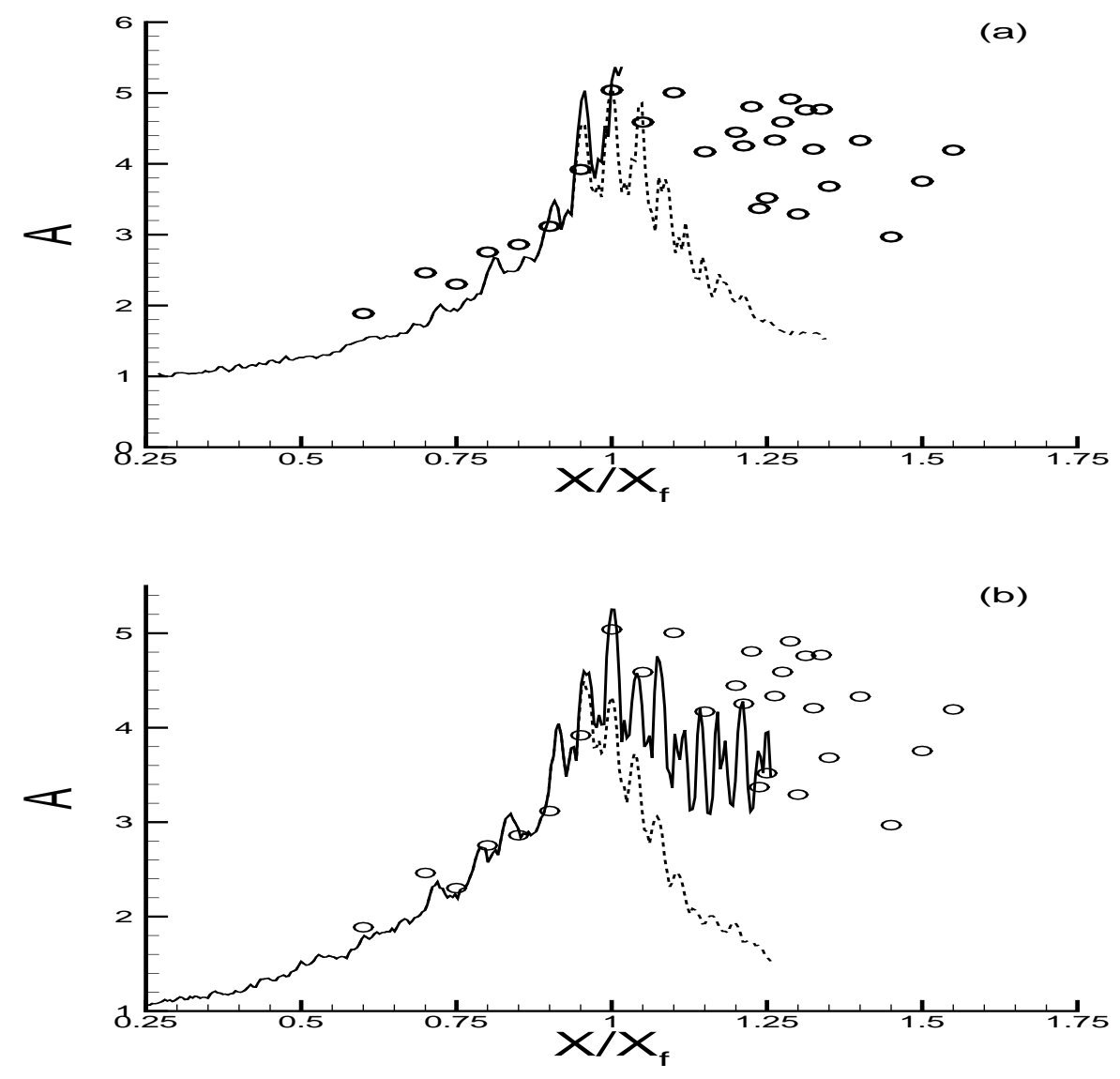

FIgURE 28. (a) Numerical (solid and dashed lines) and experimental (circle) amplification factor $A\left(X / X_{\mathrm{f}}, U\right)$ as a function of the normalized distance with wind $(U=6 \mathrm{~m} / \mathrm{s})$ for $(\partial \eta / \partial x)_{c}=0.3$ (solid line) and $(\partial \eta / \partial x)_{c}=0.4$ (dashed line) within the framework of the spatio-temporal focusing. (b) Numerical (solid and dashed lines) and experimental (circle) amplification factor $A\left(X / X_{\mathrm{f}}, U\right)$ as a function of the normalized distance in presence of wind $(U=6 \mathrm{~m} / \mathrm{s})$ and following current for $(\partial \eta / \partial x)_{c}=0.3$ (solid line) and $(\partial \eta / \partial x)_{c}=0.4$ (dashed line) within the framework of the spatio-temporal focusing.

Equations (3.12) and (3.13) are given in dimensionless form. Reference length, reference

velocity and reference pressure are, $1 / k_{0}, \sqrt{g / k_{0}}$ and $\rho_{w} g / k_{0}$ respectively.

The numerical method used to solve the evolution equations is based on a pseudo-spectral treatment with a fourth-order Runge-Kutta integrator with constant time step, similar to the method developed by Dommermuth \& Yue (1987). For more details see the paper by Skandrani et al. (1996).

It is well known that uniformly-traveling wave train of Stokes' waves are unstable to the Benjamin-Feir instability (or modulational instability) which results from a quartet resonance, that is, a resonance interaction between four components of the wave field. This instability corresponds to a quartet interaction between the fundamental component 
(the carrier) $\boldsymbol{k}_{\mathbf{0}}=k_{0}(1,0)$ counted twice and two satellites $\boldsymbol{k}_{\mathbf{1}}=k_{0}(1+p, q)$ and $\boldsymbol{k}_{\mathbf{2}}=$ $k_{0}(1-p,-q)$ where $p$ and $q$ are the longitudinal wavenumber and transversal wavenumber respectively of the modulation. Instability occurs when the following resonance conditions are fulfilled.

$$
\begin{aligned}
& \boldsymbol{k}_{\mathbf{1}}+\boldsymbol{k}_{\mathbf{2}}=2 \boldsymbol{k}_{\mathbf{0}} . \\
& \omega_{1}+\omega_{2}=2 \omega_{0} .
\end{aligned}
$$

where $\omega_{i}$ with $i=0,1,2$ are frequencies of the carrier and satellites.

A presentation of the different classes of instability of Stokes waves is given in the review paper by Dias \& Kharif (1999).

The procedure used to calculate the linear stability of Stokes waves is similar to the method described by Kharif \& Ramamonjiarisoa (1988). Let $\eta=\bar{\eta}+\eta^{\prime}$ and $\phi=\bar{\phi}+\phi^{\prime}$ be the perturbed elevation and perturbed velocity potential where $(\bar{\eta}, \bar{\phi})$ and $\left(\eta^{\prime}, \phi^{\prime}\right)$ correspond respectively to the unperturbed Stokes wave and infinitesimal perturbative motion $\left(\eta^{\prime}<<\bar{\eta}, \phi^{\prime}<<\bar{\phi}\right)$. Following Longuet-Higgins (1985), the Stokes wave of amplitude $a_{0}$ and wavenumber $k_{0}$ is computed iteratively. Substituting these decomposition in the boundary conditions linearized about the unperturbed motion and using the following forms for a two-dimensional flow:

$$
\begin{gathered}
\eta^{\prime}=\exp (\lambda t+i p x) \sum_{-\infty}^{\infty} a_{j} \exp (i j x) . \\
\left.\phi^{\prime}=\exp (\lambda t+i p x) \sum_{-\infty}^{\infty} b_{j} \exp \left(i j x+\gamma_{j} z\right)\right) .
\end{gathered}
$$

where $\lambda, a_{j}$ and $b_{j}$ are complex numbers and $\gamma_{j}=|p+j|$.

Equations (3.17) and (3.18) correspond to an eigenvalue problem for $\lambda$ with eigenvector $\boldsymbol{u}=\left(a_{j}, b_{j}\right)^{t}$ :

$$
(\boldsymbol{A}-\lambda \boldsymbol{B}) \boldsymbol{u}=0
$$

where $\boldsymbol{A}$ and $\boldsymbol{B}$ are complex matrices depending on the unperturbed wave steepness of the basic wave, $\epsilon=a_{0} k_{0}$, and the arbitrary real number $p$. The eigenvalue, $\lambda$, satisfies

$$
\operatorname{det}(\lambda \boldsymbol{B}-\boldsymbol{A})=0 .
$$

The physical disturbances are obtained from the real part of the complex expressions $\eta^{\prime}$ and $\phi^{\prime}$ at $t=0$.

McLean et al. (1981) and McLean (1982) showed that the dominant instability of a uniformly-traveling train of Stokes' waves in deep water is the two-dimensional modulational instability (class I) provided its steepness is less than $\epsilon=0.30$. For higher values of the wave steepness three-dimensional instabilities (class II) become dominant, phase locked to the unperturbed wave. Herein we shall focus on the two-dimensional nonlinear evolution of a Stokes' wave train suffering modulational instability with and without wind action. Two series of numerical simulations have been performed corresponding to two wave trains of five and nine waves respectively.

\subsubsection{Numerical simulations without wind action:}

First, we consider the case of wave trains of five waves. The initial condition is a Stokes wave of steepness $\epsilon=0.11$, disturbed by its most unstable perturbation which 
corresponds to $p \approx 0.20=1 / 5$. The fundamental wavenumber of the Stokes wave is chosen so that integral numbers of the sidebands perturbation (satellites) can be fitted into the computational domain. For $p=1 / 5$ the fundamental wave harmonic of the Stokes wave is $k_{0}=5$ and the dominant sidebands are $k_{1}=4$ and $k_{2}=6$ for subharmonic and superharmonic part of the perturbation respectively. The wave parameters have been re-scaled to have the wavelength of the perturbation equal to $2 \pi$. There exists higher harmonics present in the interactions which are not presented here. The normalized amplitude of the perturbation relative to Stokes wave amplitude is initially taken equal to $10^{-3}$. The order of nonlinearity is $M=6$, the number of mesh points is $N>(M+1) k_{\max }$ where $k_{\max }$ is the highest harmonic taken into account in the simulation. The latter criterion concerning $N$ is introduced to avoid aliasing errors. The definition of the integer $M$ can be found in Dommermuth \& Yue (1987). To compute the long time evolution of the wave train the time step $\Delta t$ is chosen equal to $T / 100$ where $T$ is the fundamental period of the basic wave. This temporal discretisation satisfies the CFL condition.

For the case without wind, the time histories of the normalized amplitude of the carrier, lower sideband and upper sideband of the most unstable perturbation are plotted in Figure 29(a). Another perturbation which was initially linearly stable becomes unstable in the vicinity of maximum of modulation resulting in the growth of the sidebands $k_{3}=3$ and $k_{4}=7$. The nonlinear evolution of the two-dimensional wave train exhibits the Fermi-Pasta-Ulam recurrence phenomenon. This phenomenon is characterized by a series of modulation-demodulation cycles in which initially uniform wave trains become modulated and then demodulated until they are again uniform. Herein one cycle is reported. At $t \approx 360 T$ the initial condition is more or less recovered. At the maximum of modulation $t=260 T$, one can observe a temporary frequency (and wavenumber) downshifting since the subharmonic mode $k_{1}=4$ is dominant. At this stage a very steep wave occurs in the group as it can be seen in Figure 30(a). Notice that the solid line represents the free surface without wind effect while the dotted line corresponds to the case with wind effect that be discussed below. Figures 30(b), 30(c) and 30(d) show the free surface profiles at several instants of time. The solid lines corresponds to the case without wind action. We can emphasize that no breaking occurs during the numerical simulation. Dold \& Peregrine (1986) have studied numerically the nonlinear evolution of different modulating wave trains towards breaking or recurrence. For a given number of waves in the wave trains breaking always occurs above a critical initial steepness, and below a recurrence towards the initial wave group is observed. This problem was revisited by Banner \& Tian (1998) who however did not considered the excitation at the maximum of modulation of the perturbation corresponding to $p=2 / 5$.

A second numerical simulation corresponding to the case of wave trains of nine waves is now considered. The initial condition is a Stokes wave of steepness $\epsilon=0.13$, disturbed by its most unstable perturbation which corresponds to $p \approx 2 / 9$. The unstable sidebands perturbation corresponding to $p=1 / 9$ is introduced as well. Hence, we consider the nonlinear evolution of the wave train when now two unstable modulations are present while in the previous case only one unstable modulation was introduced. For $p=2 / 9$ the fundamental wave harmonic of the Stokes wave is now $k_{0}=9$ and the dominant sidebands are $k_{1}=7$ and $k_{2}=11$ for subharmonic and superharmonic part of the perturbation respectively while the satellites $k_{3}=8$ and $k_{4}=10$ are the sidebands of the unstable perturbation corresponding to $p=1 / 9$. The time histories of the normalized amplitude of the carrier, lower sideband and upper sideband of the two unstable perturbations are plotted in Figure 29(b). A kind of Fermi-Pasta-Ulam recurrence can be observed which is stopped at $t \approx 500 T$ by the onset of breaking. Herein the onset of breaking is delayed by the presence of two unstable perturbations. This result is in agreement with those of Dold 

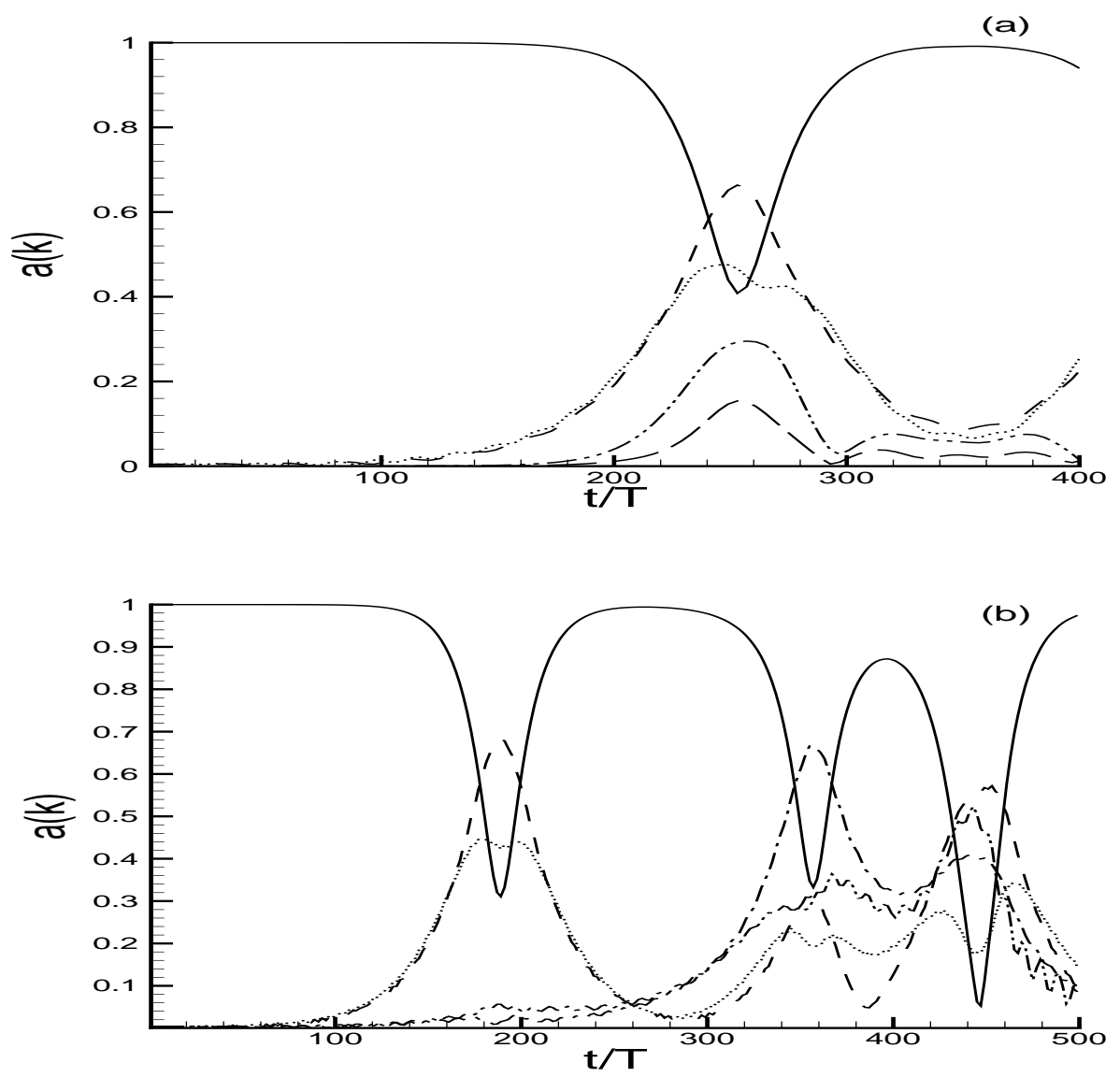

Figure 29. (a) Time histories of the amplitude of the fundamental mode, $k_{0}=5$ (solid line), subharmonic mode, $k_{1}=4$ (dashed line), and superharmonic mode, $k_{2}=6$ (dotted line), for an evolving perturbed Stokes wave of initial wave steepness $\epsilon=0.11$ and fundamental wave period $T$, without wind action. The two lowest curves (dot-dot-dashed and dot-dashed lines) correspond to the modes $k_{3}=3$ and $k_{4}=7$. (b) Time histories of the amplitude of the fundamental mode, $k_{0}=9$ (solid line), subharmonic modes, $k_{1}=7$ (dashed line) and $k_{3}=8$ (dot-dashed line), and superharmonic modes, $k_{2}=11$ (dotted line) and $k_{4}=10$ (dot-dot-dashed line), for an evolving perturbed Stokes wave of initial wave steepness $\epsilon=0.13$ and fundamental wave period $T$, without wind action.

\& Peregrine (1986) and Banner \& Tian (1998). At $t=192 T, t=360 T$ and $t=445 T$ which correspond to the first, second and third maxima of modulation without wind, a extreme wave event occurs as shown in Figures 31(a) (solid line), 31(e) and 31(f). The subharmonic sideband, $k_{1}=7$, is dominant and a temporary frequency downshifting is observed. Figures 31(b), 31(c) and 31(d) give the profiles of the wave train at $t=195 T$, $t=200 T$ and $t=210 T$ respectively.

Due to a mode competition between the satellites of the two unstable disturbances it is now the subharmonic sideband, $k_{3}=8$, of the initially less unstable perturbation which is dominant at the second maximum of modulation. 

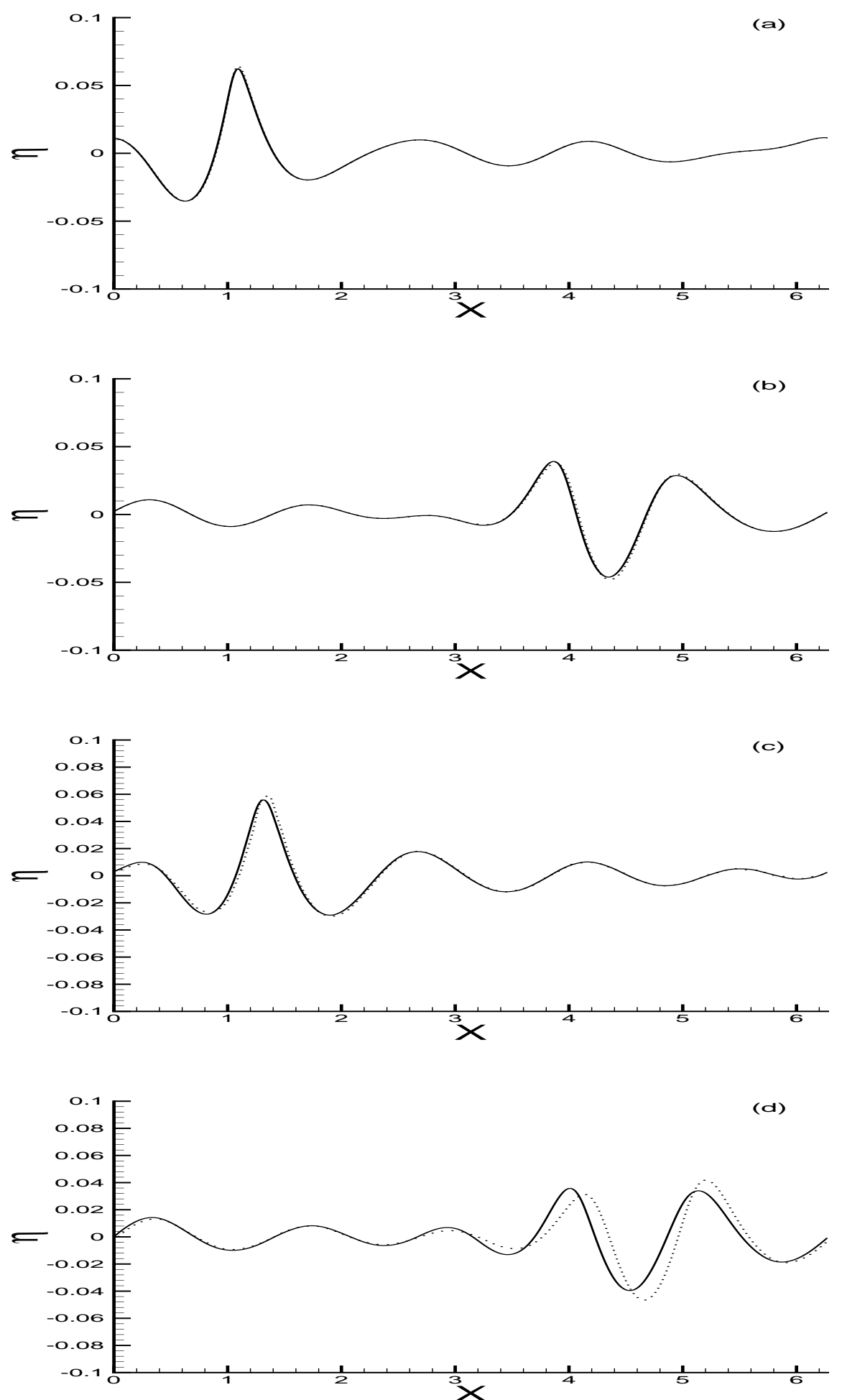

Figure 30. Surface wave profile at (a) $t=260 T$, (b) $t=265 T$, (c) $t=270 T$, (d) $t=275 T$ : without wind (solid line) and with wind (dotted line). 

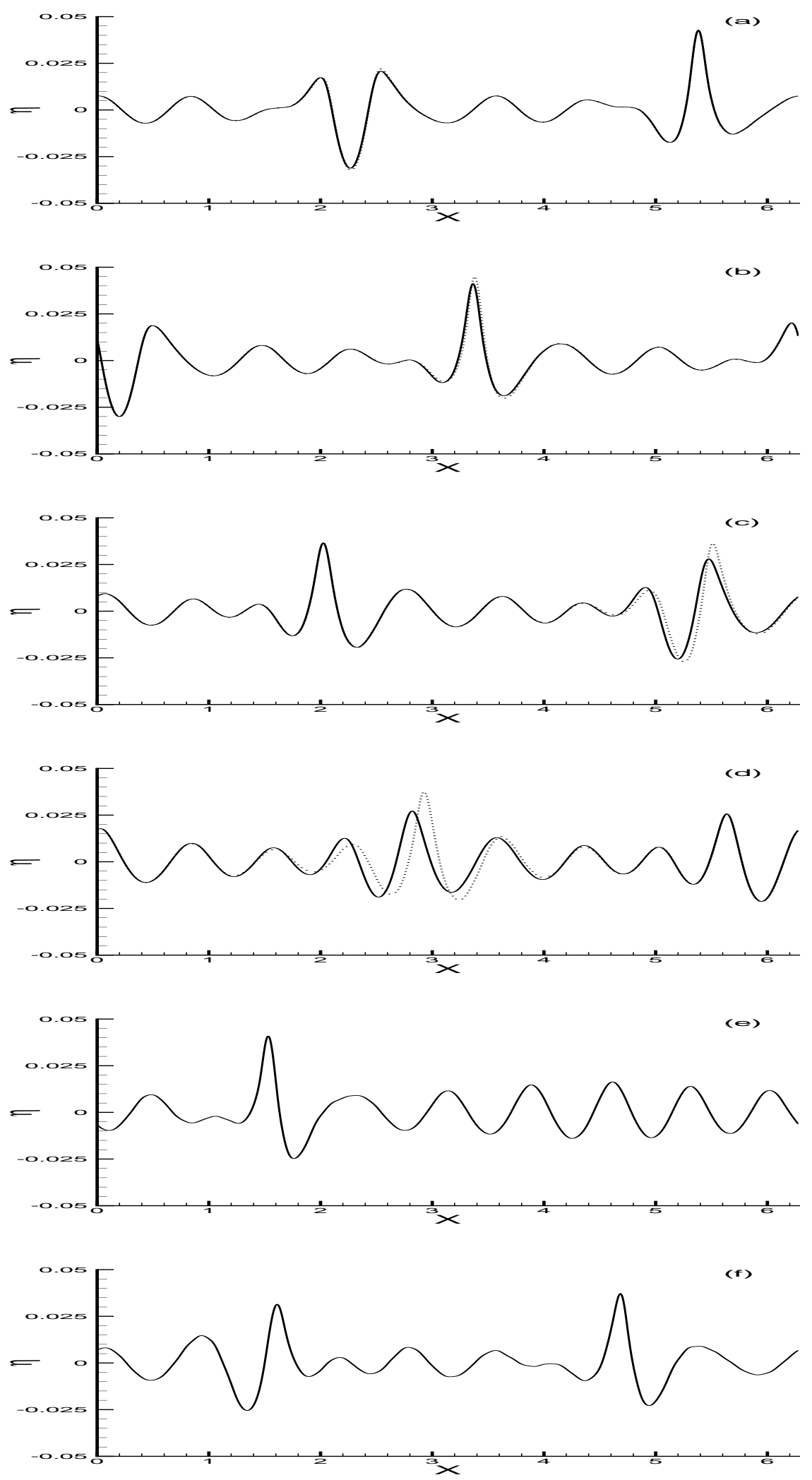

Figure 31. Surface wave profile at (a) $t=192 T$, (b) $t=195 T$, (c) $t=200 T$, (d) $t=210 T$, (e) $t=360 T$, (f) $t=445 T$ : without wind (solid line) and with wind (dotted line). 

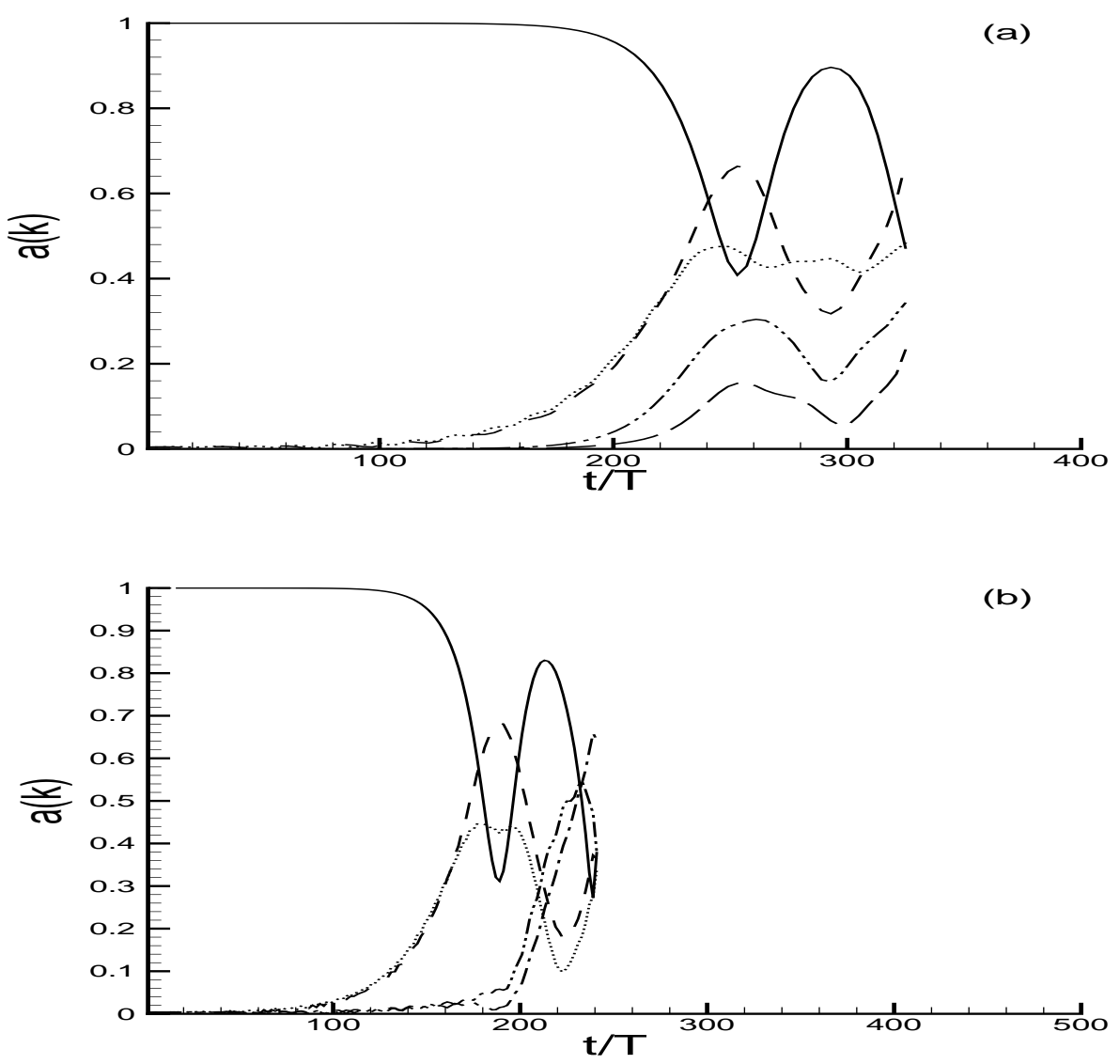

Figure 32. (a) Time histories of the amplitude of the fundamental mode, $k_{0}=5$ (solid line), subharmonic mode, $k_{1}=4$ (dashed line), and superharmonic mode, $k_{2}=6$ (dotted line), for an evolving perturbed Stokes wave of initial wave steepness $\epsilon=0.11$ and fundamental wave period $T$, with wind action $(U=1.75 c)$. The two lowest curves (dot-dot-dashed and dot-dashed lines) correspond to the modes $k_{3}=3$ and $k_{4}=7$. (b) Time histories of the amplitude of the fundamental mode, $k_{0}=9$ (solid line), subharmonic modes, $k_{1}=7$ (dashed line) and $k_{3}=8$ (dot-dashed line), and superharmonic modes, $k_{2}=11$ (dotted line) and $k_{4}=10$ (dot-dot-dashed line), for an evolving perturbed Stokes wave of initial wave steepness $\epsilon=0.13$ and fundamental wave period $T$, with wind action.

\subsubsection{Numerical simulations with wind action:}

Figures 32(a) and 32(b) are similar to Figures 29(a) and 29(b) respectively, except that now water waves evolve under wind action. Wind forcing is applied over crests of the group of five waves of slopes larger than $(\partial \eta / \partial x)_{c}=0.405$ while for the group of nine waves it is applied over crests of slope steeper than 0.5125 . These conditions are satisfied for $256 T<t<270 T$ for the first wave train and for $187 T<t<200 T$ and $237 T<t<240 T$ for the second, that is during the maximum of modulation which corresponds to the formation of the extreme wave event. When the values of the wind velocity are too high numerical simulations fail during the formation of the extreme wave event, due to breaking. During breaking wave process the slope of the surface becomes infinite, leading numerically to a spread of energy into high wavenumbers. This local 

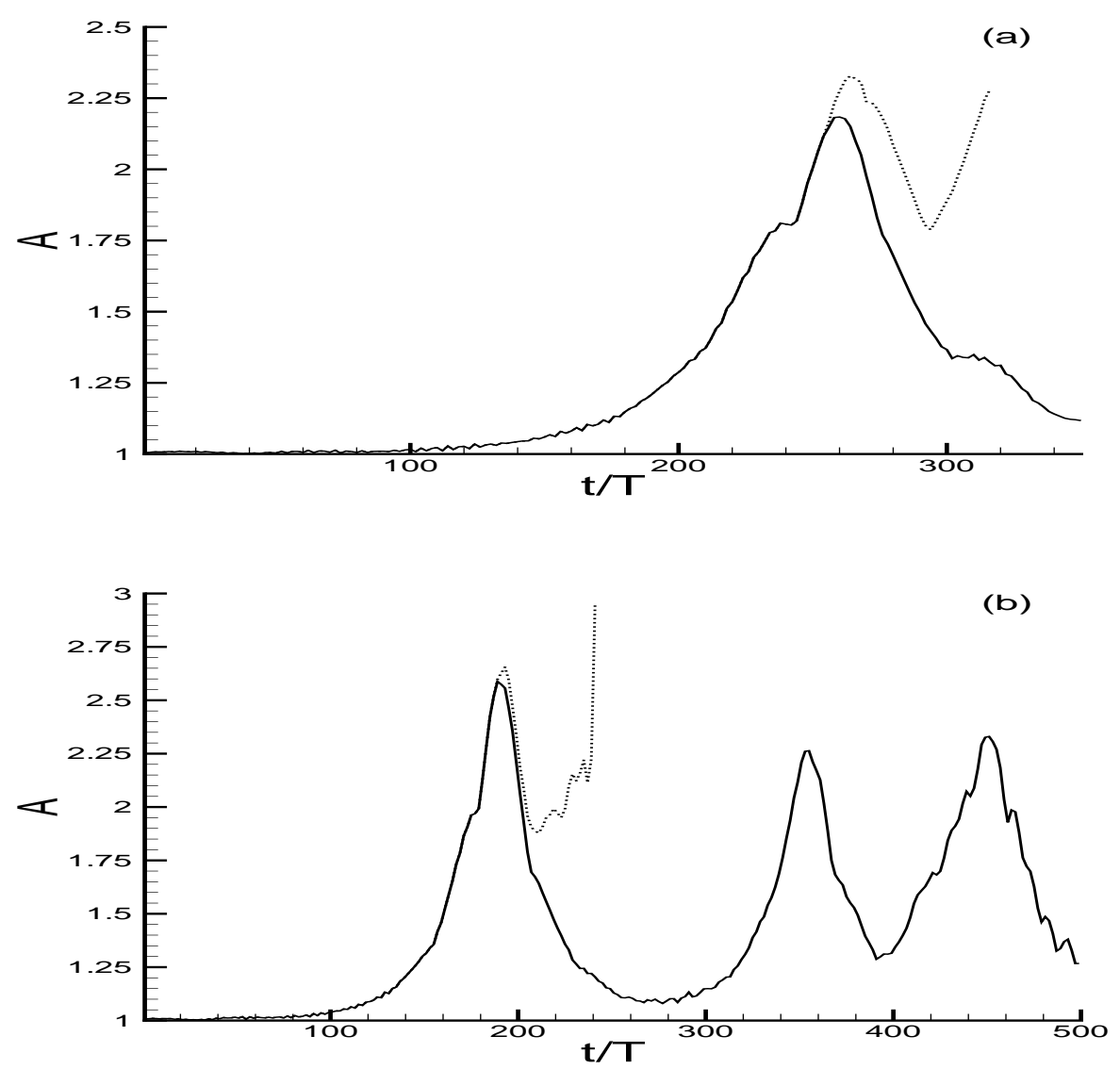

FIGURE 33. (a) Numerical amplification factor as a function of time for a wave train of five waves without wind (solid line) and with wind (dotted line) for $U=1.75 \mathrm{c}$. (b) Numerical amplification factor as a function of time for a wave train of nine waves without wind (solid line) and with wind (dashed line) for $U=1.75 \mathrm{c}$.

steepening is characterized by a numerical blow-up (for methods dealing with an Eulerian description of the flow). To avoid a too early breaking wave, the wind velocity $U$ is fixed close to $1.75 \mathrm{c}$. Owing to the weak effect of the wind on the kinematics of the crests on which it acts, the phase velocity, $c$, is computed without wind. The effect of the wind reduces significantly the demodulation cycle and thus sustains the extreme wave event. This feature is clearly shown in Figures 33(a) and 33(b) corresponding to wave trains of five and nine waves respectively. The amplification factor is stronger in presence of wind and the rogue wave criterion given by equation (1.1), $A>2.2$, is satisfied during a longer period of time. In presence of wind forcing extreme waves evolve into breaking waves at $t \approx 330 T$ and $t \approx 240 T$ for wave trains of five and nine waves respectively. For the case of a wave train of five waves, Figures 30(a), 30(b), 30(c) and 30(d) display water wave profiles at different instant of time in the vicinity of the maximum of modulation with and without wind. The solid lines corresponds to waves propagating without wind while the dotted lines represent the wave profiles under wind action. These Figures show that the wind does not modify the phase velocity of the very steep waves while it increases their height and their duration. A similar behaviour is shown in Figures 31(a), 31(b), 
$31(\mathrm{c})$ and $31(\mathrm{~d})$ corresponding to the group of nine waves. We can conclude that extreme waves occurring under wind action in both wave trains present the same features.

To summarize the results of this section, we can claim that extreme wave events generated by modulational instability in presence of wind behaves similarly to those due to dispersive spatio-temporal focusing discussed in the previous section 2 and subsection 3.3. It is found that extreme wave events generated by two different mechanisms exhibit the same behaviour in presence of wind. Furthermore, in presence of local wind forcing, extreme waves evolve to breaking waves for initial wave trains of steepness $\epsilon=0.11$ and $\epsilon=0.13$ considered herein. In another context, Banner \& Song (2002) have investigated numerically the onset and strength of breaking for deep water waves under wind forcing and surface shear. In their study wind modelling is based on the Miles theory which is different from the Jeffreys' sheltering mechanism used in this paper.

\section{Conclusions}

A series of experiments on the formation of extreme waves through the spatio-temporal focusing mechanism has been conducted in the large wind-wave tank of IRPHE and corresponding numerical simulations have been run as well. Furthermore, a second mechanism due to modulational instability and yielding to the generation of these extreme wave events has been considered numerically.

Experiments have shown that in presence of wind the kinematics and dynamics of the wave group are modified, namely the focus point is shifted downstream, the height and duration of the extreme waves are increased. A more careful and detailed analysis of the wind-wave interaction during the wave focusing emphasized the strong coupling between the wave group and the turbulent boundary layer when the extreme wave event occurs. Hence, it has been shown that air-sea fluxes are strongly enhanced in presence of strongly modulated wave groups. This strong correlation between the very steep waves of the group and the wind suggests that the Jeffreys' sheltering mechanism could be a suitable model. Indeed in presence of wind, it is shown experimentally that the occurrence of extreme wave events is accompanied by a reverse flow. Note that this mechanism which is applied only over very steep water waves works locally in space and time. For the smallest wind velocity, $U=4 \mathrm{~m} / \mathrm{s}$, considered herein, it has been shown experimentally that the wind has a sufficient aerodynamic influence to maintain extreme wave events. Nevertheless from our experiments, it is not possible to provide the value of the critical velocity for which aerodynamic influence becomes appreciable, that is, when air flow separation occurs. For $U<4 \mathrm{~m} / \mathrm{s}$, a new series of experiments is needed to determine the critical wind velocity for which air flow separation is observed. This phenomenon depends strongly on wind velocity and local wave slope as well. Numerical simulations based on two-phase flow Navier-Stokes equations and experiments are planned to investigate the occurrence of reverse air flow events as a function of both wind velocity and local wave slope.

Similar numerical simulations have been performed, corresponding to the spatio-temporal focusing studied experimentally and the wave focusing due to modulational instability as well. For the spatio-temporal focusing, a numerical wave tank has been used to generate the water waves while the Jeffreys' theory has been applied for the wind modelling to reproduce the experimental configuration. The numerical results are in qualitative good agreement with those obtained experimentally. The generation of extreme wave events due to modulational instability has concerned two numerical simulations of wave trains of 
five waves and nine waves respectively, using a pseudo-spectral method. It was found that in presence of wind, extreme wave events due to modulational instability behave similarly to those due to spatio-temporal focusing. For both cases considered in the present study, namely modulating wave trains of five and nine waves, it was found that steep waves evolve to breaking waves under local wind forcing. The role of the wind-driven current has been emphasized during extreme wave events. Following Banner \& Song (2002), it should be interesting to introduce wind forcing with surface shear instead of the uniform current used in the present investigation. Another issue is to find an indicator for the onset of rogue waves.

The present study has demonstrated that under specific conditions the modified Jeffreys'sheltering mechanism can be physically relevant to influence the dynamics of extreme wave events. The wave breaking or/and limited length of the numerical wind-wave tank do not allow this information to be readily determined and require more attention. Nevertheless, from Figure 26 one can get an estimate for $U=6 \mathrm{~m} / \mathrm{s}$ and $(\partial \eta / \partial x)_{c}=0.4$ : The duration of the extreme wave event is roughly multiplied by 1.75 . For $(\partial \eta / \partial x)_{c}=0.3$ we observe a blow up of the numerical simulation that corresponds to breaking. On the other hand when a co-flowing current is introduced no breaking occurs and the extreme wave event is sustained longer. In our numerical experiments, the normalized amplitude does not become less than 2.2 beyond the maximum of modulation (see Figure 28-b).

We are grateful to the referees for their useful comments that help us to improve the paper. EP gratefully acknowledges the Centre National de la Recherche Scientifique which supported his stay at the Institut de Recherche sur les Phénomènes Hors Equilibre as Directeur de Recherche. This ressearch was partly supported by INTAS (06-1000013-9236).

\section{REFERENCES}

BALK, A.M. 1996 The suppression of short waves by a train of long waves. J. Fluid Mech. 315, $139-150$.

BANNER, M.I. 1990 The influence of wave breaking on the surface distribution in wind-wave interactions. J. Fluid Mech. 211, 463-495.

Banner, M.I. \& Melville, W.K. 1976 On the separation of air flow over water waves. J. Fluid Mech. 77, 825-842.

BAnner, M.I. \& Song, J. 2002 On determining the onset and strength of breaking for deep water waves. part ii: Influence of wind forcing and surface shear. J. Phys. Oceanogr. 32 (9), 2559-2570.

BAnNeR, M.I. \& Tian, X. 1998 On the determination of the onset of breaking for modulating surface gravity water waves. J. Fluid Mech. 367, 107-137.

Benjamin, T.B. \& Feir, J.E. 1967 The desintegration of wave trains on deep water. part 1. theory. J. Fluid Mech. 27, 417-430.

Bliven, L.F., Huang, N.E. \& Long, S.R. 1986 Experimental study of the influence of wind on benjamin-feir sideband instability. J. Fluid Mech. 162, 237-260.

Calini, A. \& Schober, C.M. 2002 Homoclinic chaos increases the likelihood of rogue wave formation. Phys. Lett. A 298, 335-349.

Clamond, D., Francius, M., Grue, J. \& Kharif, C. 2006 Strong interaction between envelope solitary surface gravity waves. Eur. J. Mech. B/Fluids 25 (5), 536-553.

Dias, F. \& KhARIF, C. 1999 Nonlinear gravity and capillary-gravity waves. Annu. Rev. Fluid Mech. 31, 301-346.

Dold, J.W. \& Peregrine, D.H. 1986 Water wave modulation. In Proc. 20th Int. Conf. Coastal Engng., , vol. 1, pp. 163-175. ASCE, Taipei.

Dommermuth, D.G. \& Yue, D.K.P. 1987 A high-order spectral method for the study of nonlinear gravity waves. J. Fluid Mech. 184, 267-288. 
Dyachenko, A.I. \& Zakharov, V.E. 2005 Modulational instability of stokes wave $\rightarrow$ freak wave. J. Exp. Theor. Phys. 81 (6), 318-322.

Dysthe, K.B. 2001 Modelling a "Rogue Wave" - Speculations or a realistic possibility? In Rogues Waves 2000 (ed. M. Olagnon \& G.A. Athanassoulis), , vol. 32, pp. 255-264. Ifremer, Brest.

Dysthe, K.B. \& Trulsen, K. 1999 Note on breather type solutions of the nls as a model for freak waves. Phys. Scripta 82, 48-52.

Giovanangeli, J.P. 1988 A new method for measuring static pressure fluctuations with application to wind wave interaction. Exp. Fluids 6, 1221-1225.

Giovanangeli, J.P. \& Chambaud, P. 1987 Pressure, velocity and temperature sensitivities of a bleed-type pressure sensor. Rev. Sci. Instrum. 58, 154-164.

Giovanangeli, J.P., Kharif, C. \& Pelinovsky, E. 2005 Experimental study of the wind effect on the focusing of transient wave groups. In Rogues Waves 2004 (ed. M. Olagnon \& M. Prevosto). Ifremer, Brest.

Henderson, K.L., Peregrine, D.H. \& Dold, J.W. 1999 Unsteady water wave modulations: Fully nonlinear solutions and comparison with the nonlinear schrödinger equation. Wave Motion 29, 341-361.

Janssen, P.A.E.M. 2003 Nonlinear four-wave interactions and freak waves. J. Phys. Oceanogr. 33, $863-884$

Jeffreys, H. 1925 On the formation of wave by wind. Proc. Roy. Soc. A 107, 189-206.

KaWAI, S. 1982 Structure of air flow separation over wind wave crests. Boundary-Layer Mteoro. 23 (4), 503-521.

Kharif, C. \& Pelinovsky, E. 2003 Physical mechanisms of the rogue wave phenomenon. Eur. J. Mech. B/Fluids 22, 603-634.

Kharif, C., Pelinovsky, E., Talipova, T. \& Slunyaev, A. 2001 Focusing of nonlinear wave groups in deep water. J. Exp. Theor. Phys. Lett. 73(4), 170-175.

Kharif, C. \& RamamonjiarisoA, A. 1988 Deep water gravity wave instabilities at large steepness. Phys. Fluids 31, 1286-1288.

LATIF, M.A. 1974 Acoustic effects on pressure measurements over water waves in the laboratory. Tech. Rep. 25. Coastal and Oceanographic Engineering Laboratory, Gainsville, Florida.

Li, J.C., Hui, W.H. \& Donelan, M.A. 1987 Effects of velocity shear on the stability of surface deep water wave trains. In Nonlinear Water Waves (IUTAM Symp.), pp. 213-220. Springer Verlag.

Longuet-Higgins, M.S. 1985 Bifurcation in gravity waves. J. Fluid Mech. 151, 457-475.

Mastenbroeck, C., Makin, V.K., Garat, M.H. \& Giovanangeli, JP 1996 Experimental evidence of the rapid distorsion of turbulence in the air flow over water waves. J. Fluid Mech. 318, 273-1302.

MCLEan, J.W. 1982 Instabilities of finite-amplitude water waves. J. Fluid Mech. 114, 315-330.

Mclean, J.W., Ma, Y.C., Martin, D.U., Saffman, P.G. \& Yuen, H.C 1981 Threedimensional instability of finite-amplitude water waves. Phys. Rev. Lett. 46, 817-820.

N. Reul, H. Branger \& Giovanangeli, J.-P. 1999 Air flow separationover unsteady breaking waves. Phys. Fluids 11, 1959-1961.

Osborne, A.R., Onorato, M. \& Serio, M. 2000 The nonlinear dynamics of rogue waves and holes in deep-water gravity wave train. Phys. Rev. A 275, 386-393.

Papadimitrakis, Y.A., Hsu, Y. \& Street, R.L. 1986 The role of wave-induced pressure fluctuations in the transfer accross an air-water interface. J. Fluid Mech. 170, 113-127.

Pelinovsky, E., Talipova, T. \& Kharif, C. 2000 Nonlinear dispersive mechanism of the freak wave formation in shallow water. Physica D 147(1-2), 83-94.

Skandrani, C., Kharif, C. \& Poitevin, J. 1996 Nonlinear evolution of water surface waves: The frequency downshifting phenomenon. Contemp. Math. 200, 157-171.

Torrence, C. \& Compo, G.P. 1998 A practical guide to wavelet analysis. Bull. of the American Meteorol. Soc. $\mathbf{7 9 ( 1 )}, 61-78$.

Touboul, J., Giovanangeli, J.P., Kharif, C. \& Pelinovsky, E. 2006 Freak waves under the action of wind: Experiments and simulations. Eur. J. Mech. B/Fluids 25 (5), 662-676.

Touboul, J., Pelinovsly, E. \& Kharif, C. 2007 Nonlinear focusing wave groups on current. J. Exp. Theor. Phys., Submitted . 
WASEDA, T. \& Tulin, M.P. 1999 Experimental study of the stability of deep-water wave trains including wind effects. J. Fluid Mech. 401, 55-84.

Whitham, G.B. 1967 Nonlinear dispersion of water waves. J. Fluid Mech. 27, 399-412.

ZaKharov, V.E. 1968 Stability of periodic waves of finite amplitude on the surface of deep water. J. Appl. Mech. Tech. Phys. 9, 190-194. 